\title{
Identification and characterization of chestnut branch-inhabiting melanocratic fungi in China
}

\author{
Jiang $\mathrm{N}^{1}, \mathrm{Li} \mathrm{J}^{2}$, Piao $\mathrm{CG}^{3}$, Guo MW ${ }^{3}$ and Tian $\mathrm{CM}^{1^{*}}$ \\ ${ }^{I}$ The Key Laboratory for Silviculture and Conservation of Ministry of Education, Beijing Forestry University, Beijing \\ 100083, China \\ ${ }^{2}$ General Station of Forest Pest Management, State Forestry Administration, Shenyang 110034, China \\ ${ }^{3}$ China Forestry Culture Collection Center, Chinese Academy of Forestry, Beijing 100091, China
}

Jiang N, Li J, Piao CG, Guo MW, Tian CM 2018 - Identification and characterization of chestnut branch-inhabiting melanocratic fungi in China. Mycosphere 9(6), 1268-1289, Doi 10.5943/mycosphere/9/6/14

\begin{abstract}
Chinese chestnut (Castanea mollissima) is an important commercial tree species widely cultivated in most provinces in China. Chestnut branch-inhabiting fungi may be responsible for branch canker and dieback. In present study, dead corticated chestnut branches with superficial ascomata or conidiomata were collected from chestnut plantations in Hebei, Shaanxi and Shandong provinces. Fungi producing melanocratic ascospores or conidia were observed and identified based on both morphology and analyses of DNA sequence data. Specimens were identified to five species, viz. Neopseudomelanconis castaneae gen. et sp. nov., Aplosporella javeedii Jami, Gryzenh., Slippers and M.J. Wingf., Coryneum umbonatum Nees, Lopadostoma americanum Jaklitsch, J. Fourn., J.D. Rogers and Voglmayr and Myrmaecium fulvopruinatum Jaklitsch and Voglmayr.
\end{abstract}

Key words - 2 new taxa - canker - Diaporthales - Pseudomelanconidaceae - taxonomy

\section{Introduction}

Chestnuts are important fruit and timber trees worldwide. The four main species are commonly known as American, Chinese, European and Japanese chestnuts. Chinese sweet chestnut (Castanea mollissima Blume) is widely cultivated in China for its important economic value (Zhang et al. 2009, Lu \& Guo 2017). Nevertheless, fungal diseases have greatly disturbed the healthy growth of chestnut (Tai 1979, Visentin et al. 2012, Gong et al. 2017, Jiang et al. 2018). Chestnut blight caused by Cryphonectria parasitica is the most notorious branch canker disease among various chestnut diseases worldwide (Jiang et al. 2018, Rigling \& Prospero 2018).

Melanocratic fungi are an artificial taxonomic group which produces melanocratic conidia or ascospores (Wijayawardene et al. 2016). They are more recognizable than species which produce hyaline spores, hence we started our taxonomic work on branch-inhabiting fungi on Castanea mollissima with melanocratic taxa (Fig. 1). In this study, fresh specimens with melanocratic spores were collected from Hebei, Shaanxi and Shandong provinces in China, and were identified to five genera, viz. Aplosporella, Coryneum, Lopadostoma, Myrmaecium and Neopseudomelanconis gen. nov., with evidence from morphology and phylogenies.

Branch-inhabiting fungi on Castanea mollissima mainly contain canker pathogens and 
saprobes. For example, Cryphonectria parasitica is an important canker pathogen and C. radicalis is considered as saprobe on chestnut trees (Gryzenhout et al. 2009). Determining whether fungi are parasitic or saprobic depends on their pathogenicity to their hosts, hence there is no absolute limit between pathogens and saprobes. In China, identification of branch-inhabiting pathogens and saprobes on chestnut is poor. Therefore, the present study was conducted (i) to identify branchinhabiting melanocratic fungi on chestnut trees and (ii) to propose Neopseudomelanconis gen. nov. based on both morphological and phylogenetic evidences.
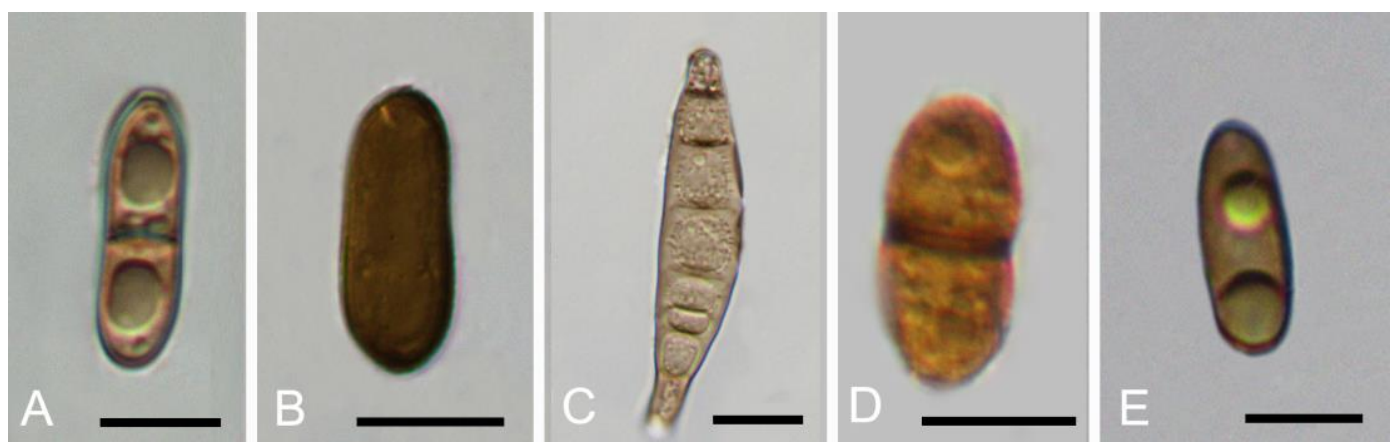

Figure 1 - Melanocratic spores observed from Castanea mollissima. A Neopseudomelanconis castaneae. B Aplosporella javeedii. C Coryneum umbonatum. D Myrmaecium fulvopruinatum. E Lopadostoma americanum. Scale bars: $\mathrm{A}-\mathrm{E}=10 \mu \mathrm{m}$.

\section{Materials \& Methods}

\section{Isolates and morphology}

Fresh specimens of dead corticated branches were collected from Castanea mollissima in Hebei, Shaanxi and Shandong provinces in China. Single conidial and ascosporous isolates were established by removing a mucoid spore mass from conidiomata or ascomata, and spreading the suspension on the surface of PDA (potato dextrose agar) or MEA (malt extract agar). After inoculation, agar plates were incubated at $25{ }^{\circ} \mathrm{C}$ to induce germination of spores. Single germinating spores were then transferred to clean plates under a dissecting microscope with a sterile needle (Jaklitsch et al. 2014, 2015, Jami et al. 2014, Wijayawardene et al. 2016, Senanayake et al. 2017). Specimens and isolates were deposited in the Museum of Beijing Forestry University (BJFC). Axenic cultures are maintained in the China Forestry Culture Collection Center (CFCC).

Species identification was based on morphological features of the conidiomata or ascomata produced on infected chestnut branches. Cross-sections were prepared by hand using a double-edge blade under a Leica stereomicroscope (M205 FA). At least 20 conidiomata/ascomata, 10 asci and 50 conidia/ascospores were measured to calculate the mean size and standard deviation. Measurements were reported as maxima and minima in parentheses and the range representing the mean plus and minus the standard deviation of the number of measurements given in parentheses (Voglmayr et al. 2017). Microscopic photographs were captured with a Nikon Eclipse 80i microscope equipped with a Nikon digital sight DS-Ri2 high definition colour camera, using differential interference contrast (DIC) illumination and the Nikon software NIS-Elements D Package v. 3.00. Cultural characteristics of isolates incubated on PDA or MEA in the dark at $25^{\circ} \mathrm{C}$ were recorded.

\section{Molecular characterization and multi-locus phylogenetic analysis}

Genomic DNA was extracted from axenic living cultures with cellophane using a modified CTAB method (Doyle \& Doyle 1990). To amplify the internal transcribed spacer (ITS), large subunit (LSU), translation elongation factor $1 \alpha$ (TEF1- $\alpha$ ) and the largest subunit of RNA polymerase II (RPB2), we used the primers LR0R and LR5 (Moncalvo et al. 1995, Vilgalys \& Hester 1990), ITS1 and ITS4 (White et al. 1990), EF1-688F and EF1-986R (Alves et al. 2008, 
Carbone \& Kohn 1999), dRPB2-5f and dRPB2-7r (Voglmayr et al. 2016), respectively. The polymerase chain reaction assay was conducted as described by Fan et al. (2018). The PCR amplification products were estimated visually by electrophoresis in $2 \%$ agarose gels. DNA sequencing was performed using an ABI PRISM ${ }^{\circledR}$ 3730XL DNA Analyzer with BigDye ${ }^{\circledR}$ Terminater Kit v.3.1 (Invitrogen) at the Shanghai Invitrogen Biological Technology Company Limited (Beijing, China).

Sequences from this study and reference sequences obtained from GenBank (Tables 1-4) were aligned and edited manually using MEGA6 (Tamura et al. 2013). The alignments were concatenated for phylogenetic analyses. Maximum parsimony (MP) analysis was conducted with PAUP v.4.0b10 (Swofford 2003), maximum likelihood (ML) analysis with PhyML v.3.1 (Guindon et al. 2010) and Bayesian inference (BI) with MrBayes v.3.1.2 (Ronquist \& Huelsenbeck 2003). Sequences data were deposited in GenBank (Tables 1-4). The multilocus file was deposited in TreeBASE (www.treebase.org) as accession S22783. Introduction of the new species based on molecular data follow the recommendations of Jeewon \& Hyde (2016).

Table 1 Strains of Diaporthales species used in the molecular analyses in this study, the genes sequenced and GenBank accessions. The new strains from the current study are in red.

\begin{tabular}{|c|c|c|c|c|}
\hline \multirow{2}{*}{ Species } & \multirow{2}{*}{ Strain/Specimen } & \multirow{2}{*}{ Host } & \multicolumn{2}{|c|}{ GenBank numbers } \\
\hline & & & ITS & LSU \\
\hline Apiosporopsis carpinea & CBS 771.79 & Carpinus betulus & NA & AF277130 \\
\hline Apiosporopsis sp. & Masuya 11Af2-1 & Alnus firma & NA & AB669034 \\
\hline Apoharknessia insueta & CBS 111377 & Eucalyptus pellita & JQ706083 & AY720814 \\
\hline $\begin{array}{l}\text { Asterosporium } \\
\text { asterospermum }\end{array}$ & MFLU 15-3555 & Fagus sylvatica & NA & MF190062 \\
\hline $\begin{array}{l}\text { Auratiopycnidiella } \\
\text { tristaniopsidis }\end{array}$ & CBS 132180 & Tristaniopsis laurina & JQ685516 & JQ685522 \\
\hline $\begin{array}{l}\text { Chiangraiomyces } \\
\text { bauhiniae }\end{array}$ & MFLUCC 17-1669 & Bauhinia sp. & MF190118 & MF190064 \\
\hline Coniella straminea & CBS 149.22 & Fragaria sp. & AY339348 & AF362569 \\
\hline Coniella wangiensis & CBS 132530 & Eucalyptus sp. & JX069873 & JX069857 \\
\hline Coryneum arausiacum & MFLUCC 13-0658 & Quercus sp. & MF190120 & MF190066 \\
\hline Coryneum arausiacum & MFLUCC 15-1110 & Quercus sp. & MF190121 & MF190067 \\
\hline Coryneum modonium & AR 3558 & Castanea sativa & NA & EU683073 \\
\hline Coryneum depressum & AR 3897 & Quercus cerris & NA & EU683074 \\
\hline Coryneum umbonatum & AR 3541 & Quercus cerris & NA & EU683072 \\
\hline $\begin{array}{l}\text { Cryphonectria } \\
\text { macrospora }\end{array}$ & CBS 122593 & Quercus mongolica & EU199182 & AF408340 \\
\hline Cryphonectria parasitica & ATCC 38755 & Castanea dentata & AY141856 & EU199123 \\
\hline $\begin{array}{l}\text { Cryptosporella } \\
\text { hypodermia }\end{array}$ & AR 3552 & Ulmus minor & EU199181 & AF408346 \\
\hline Cytospora chrysosperma & CFCC 89600 & Sophora japonica & KR045623 & KR045623 \\
\hline Dendrostoma mali & CFCC 52102 & Malus spectabilis & MG682072 & MG682012 \\
\hline Diaporthe eres & CBS 109767 & Acer campestre & KC343075 & AF408350 \\
\hline $\begin{array}{l}\text { Diaporthosporella } \\
\text { cercidicola }\end{array}$ & CFCC 51994 & Cercis chinensis & KY852492 & KY852515 \\
\hline Diaporthostoma machili & CFCC 52100 & Machilus leptophylla & MG682080 & MG682020 \\
\hline Disculoides eucalypti & CPC 17650 & Eucalyptus sp. & JQ685517 & JQ685523 \\
\hline Ditopella ditopa & CBS 109748 & Alnus glutinosa & EU199187 & EU199126 \\
\hline Erythrogloeum hymenaeae & СРC 18819 & Hymenaea courbaril & JQ685519 & JQ685525 \\
\hline Gnomonia gnomon & CBS 199.53 & Corylus avellana & AY818956 & AF408361 \\
\hline
\end{tabular}


Table 1 Continued.

\begin{tabular}{|c|c|c|c|c|}
\hline \multirow[b]{2}{*}{ Species } & \multirow[b]{2}{*}{ Strain/Specimen } & \multirow[b]{2}{*}{ Host } & \multicolumn{2}{|c|}{ GenBank numbers } \\
\hline & & & ITS & $\mathbf{L S U}$ \\
\hline Harknessia eucalypti & CBS 342.97 & Eucalyptus regnans & AY720745 & AF408363 \\
\hline Harknessia molokaiensis & CBS 109779 & Eucalyptus robusta & NA & AF408390 \\
\hline Hercospora tiliae & CBS 109746 & Tilia tomentosa & NA & AF408365 \\
\hline Juglanconis appendiculata & D96 & Juglans nigra & KY427139 & KY427139 \\
\hline Juglanconis juglandina & ME23 & Juglans nigra & KY427150 & KY427150 \\
\hline Lamproconium desmazieri & MFLUCC 15-0870 & Tilia tomentosa & KX430134 & KX430135 \\
\hline Lasmenia sp. & CBS 124123 & $\begin{array}{l}\text { Nephelium } \\
\text { lappaceum }\end{array}$ & GU797406 & JF838338 \\
\hline Macrohilum eucalypti & СРC 10945 & Eucalyptus sp. & DQ195781 & DQ195793 \\
\hline Melanconiella ellisii & BPI 878343 & Carpinus caroliniana & JQ926271 & JQ926271 \\
\hline Melanconiella spodiaea & MSH & Carpinus betulus & JQ926298 & JQ926298 \\
\hline Melanconis betulae & CFCC 50471 & Betula albosinensis & KT732952 & KT732971 \\
\hline Melanconis stilbostoma & CFCC 50475 & Betula platyphylla & KT732956 & KT732975 \\
\hline Nakataea oryzae & CBS 243.76 & NA & KM484861 & DQ341498 \\
\hline $\begin{array}{l}\text { Neopseudomelanconis } \\
\text { castaneae }\end{array}$ & CFCC 52787 & Castanea mollissima & MH469162 & MH469164 \\
\hline $\begin{array}{l}\text { Neopseudomelanconis } \\
\text { castaneae }\end{array}$ & CFCC 52788 & Castanea mollissima & MH469163 & MH469165 \\
\hline Pachytrype princeps & Rogers S & NA & NA & FJ532382 \\
\hline Paradiaporthe artemisiae & MFLUCC 14-0850 & Artemisia sp. & MF190155 & MF190100 \\
\hline Prosopidicola mexicana & CBS 113530 & Prosopis glandulosa & AY720710 & NA \\
\hline Pseudomelanconis caryae & CFCC 52110 & Carya cathayensis & MG682082 & MG682022 \\
\hline $\begin{array}{l}\text { Pseudoplagiostoma } \\
\text { eucalypti }\end{array}$ & CBS 124807 & Eucalyptus urophylla & GU973512 & GU973606 \\
\hline Pseudoplagiostoma oldii & CBS 115722 & $\begin{array}{l}\text { Eucalyptus } \\
\text { camaldulensis }\end{array}$ & GU973535 & GU973610 \\
\hline Pyricularia grisea & Ina168 & NA & AB026819 & AB026819 \\
\hline Rossmania ukurunduensis & AR 3484 & Acer ukurunduense & NA & EU683075 \\
\hline Stegonsporium pyriforme & CBS 124487 & Acer heldreichii & KF570160 & KF570160 \\
\hline Stilbospora macrosperma & CBS 121883 & Carpinus betulus & JX517290 & JX517299 \\
\hline Sydowiella fenestrans & CBS 125530 & $\begin{array}{l}\text { Chamerion } \\
\text { angustifolium }\end{array}$ & JF681956 & EU683078 \\
\hline Synnemasporella aculeans & CFCC 52094 & Rhus chinensis & MG682086 & MG682026 \\
\hline $\begin{array}{l}\text { Synnemasporella } \\
\text { toxicodendri }\end{array}$ & CFCC 52097 & $\begin{array}{l}\text { Toxicodendron } \\
\text { sylvestre }\end{array}$ & MG682089 & MG682029 \\
\hline
\end{tabular}

Table 2 Strains of Aplosporella species used in the molecular analyses in this study, the genes sequenced and GenBank accessions. The new strains from the current study are in red.

\begin{tabular}{lllll}
\hline \multirow{2}{*}{ Species } & \multirow{2}{*}{ Strain/Specimen } & Host & \multicolumn{2}{l}{ GenBank numbers } \\
\cline { 4 - 5 } & & & ITS & TEF1-a \\
\hline Aplosporella africana & CBS 121777 & Acacia mellifera & EU101315 & EU101360 \\
Aplosporella africana & CBS 121779 & Acacia mellifera & EU101317 & EU101362 \\
Aplosporella artocarpi & CPC 22791 & Artocarpus heterophyllus & KM006450 & KM006481 \\
Aplosporella hesperidica & CBS 208.37 & NA & JX681069 & NA \\
Aplosporella javeedii & CFCC 50052 & Gleditsia sinensis & KP208838 & KP208844 \\
\hline
\end{tabular}


Table 2 Continued.

\begin{tabular}{|c|c|c|c|c|}
\hline \multirow[b]{2}{*}{ Species } & \multirow[b]{2}{*}{ Strain/Specimen } & \multirow[b]{2}{*}{ Host } & \multicolumn{2}{|c|}{ GenBank numbers } \\
\hline & & & ITS & TEF1- $\alpha$ \\
\hline Aplosporella javeedii & CFCC 50053 & Sophora japonica & KP208839 & KP208845 \\
\hline Aplosporella javeedii & CFCC 52777 & Castanea mollissima & MH458901 & MH482840 \\
\hline Aplosporella ginkgonis & CFCC 89660 & Morus alba & KR045623 & KP310847 \\
\hline Aplosporella ginkgonis & CFCC 89661 & Ginkgo biloba & KM030583 & KM030597 \\
\hline $\begin{array}{l}\text { Aplosporella } \\
\text { macropycnidia }\end{array}$ & CGMCC3.17725 & Cerasus yedoensis & KT343648 & KX011176 \\
\hline $\begin{array}{l}\text { Aplosporella } \\
\text { macropycnidia }\end{array}$ & CGMCC3.17726 & Cerasus yedoensis & KT343649 & KX011177 \\
\hline Aplosporella papillata & CBS 121780 & Acacia tortillas & EU101328 & EU101373 \\
\hline Aplosporella papillata & CBS 121781 & Acacia erioloba & EU101329 & EU101374 \\
\hline Aplosporella prunicola & CBS 121167 & $\begin{array}{l}\text { Prunus persica var. } \\
\text { nucipersica }\end{array}$ & KF766147 & NA \\
\hline Aplosporella prunicola & STE-U 6326 & $\begin{array}{l}\text { Prunus persica var. } \\
\text { nucipersica }\end{array}$ & EF564375 & NA \\
\hline Aplosporella thailandica & MFLU 16-0615 & NA & KX423536 & KX423537 \\
\hline Aplosporella yalgorensis & MUCC 511 & Eucalyptus gomphocephala & EF591926 & EF591977 \\
\hline Aplosporella yalgorensis & MUCC 512 & Mimetes cucullata & EF591927 & EF591978 \\
\hline Saccharata proteae & CBS 115206 & NA & KC343004 & KC343730 \\
\hline
\end{tabular}

Table 3 Strains of Lopadostoma species used in the molecular analyses in this study, the genes sequenced and GenBank accessions. The new strains from the current study are in red.

\begin{tabular}{|c|c|c|c|c|c|}
\hline \multirow{2}{*}{ Species } & \multirow{2}{*}{ Strain/Specimen } & \multirow{2}{*}{ Host } & \multicolumn{3}{|c|}{ GenBank numbers } \\
\hline & & & ITS & LSU & RPB2 \\
\hline $\begin{array}{l}\text { Lopadostoma } \\
\text { americanum }\end{array}$ & CBS 133211 & Quercus sp. & KC774568 & KC774568 & KC774525 \\
\hline $\begin{array}{l}\text { Lopadostoma } \\
\text { americanum }\end{array}$ & CFCC 52313 & $\begin{array}{l}\text { Castanea } \\
\text { mollissima }\end{array}$ & MH454484 & MH454486 & MH482841 \\
\hline $\begin{array}{l}\text { Lopadostoma } \\
\text { americanum }\end{array}$ & CFCC 52314 & $\begin{array}{l}\text { Castanea } \\
\text { mollissima }\end{array}$ & MH454485 & MH454487 & MH482842 \\
\hline $\begin{array}{l}\text { Lopadostoma } \mathrm{cf} . \\
\text { amoenum }\end{array}$ & MUCL 51842 & Fagus sylvatica & KC774569 & KC774569 & NA \\
\hline Lopadostoma dryophilum & CBS 133213 & Quercus petraea & KC774570 & KC774570 & KC774526 \\
\hline Lopadostoma dryophilum & LG23 & Quercus petraea & KC774571 & KC774571 & KC774527 \\
\hline Lopadostoma dryophilum & LG24 & Quercus petraea & KC774572 & KC774572 & KC774528 \\
\hline Lopadostoma fagi & $\mathrm{LF}$ & NA & KC774574 & KC774574 & KC774530 \\
\hline Lopadostoma fagi & CBS 133206 & Fagus sylvatica & KC774575 & KC774575 & KC774531 \\
\hline Lopadostoma fagi & LF2 & Fagus sylvatica & KC774576 & KC774576 & KC774532 \\
\hline Lopadostoma gastrinum & LG1 & Carpinus betulus & KC774579 & KC774579 & KC774535 \\
\hline Lopadostoma gastrinum & CBS 133210 & Ulmus glabra & KC774581 & KC774581 & KC774536 \\
\hline Lopadostoma gastrinum & CBS 134632 & Ulmus minor & KC774584 & KC774584 & KC774537 \\
\hline Lopadostoma insulare & LG32 & Quercus coccifera & KC774588 & KC774588 & KC774541 \\
\hline Lopadostoma insulare & CBS 133214 & Quercus ilex & KC774589 & KC774589 & KC774542 \\
\hline Lopadostoma lechatii & CBS 133694 & Carpinus betulus & KC774590 & KC774590 & KC774543 \\
\hline $\begin{array}{l}\text { Lopadostoma } \\
\text { linospermum }\end{array}$ & CBS 133208 & Pistacia lentiscus & KC774591 & KC774591 & KC774544 \\
\hline
\end{tabular}


Table 3 Continued.

\begin{tabular}{|c|c|c|c|c|c|}
\hline \multirow{2}{*}{ Species } & \multirow{2}{*}{ Strain/Specimen } & \multirow{2}{*}{ Host } & \multicolumn{3}{|c|}{ GenBank numbers } \\
\hline & & & ITS & LSU & RPB2 \\
\hline $\begin{array}{l}\text { Lopadostoma } \\
\text { linospermum }\end{array}$ & LPL1 & Pistacia lentiscus & KC774592 & KC774592 & KC774545 \\
\hline $\begin{array}{l}\text { Lopadostoma } \\
\text { meridionale }\end{array}$ & CBS 133209 & Quercus ilex & KC774593 & KC774593 & KC774546 \\
\hline $\begin{array}{l}\text { Lopadostoma } \\
\text { meridionale }\end{array}$ & LG29 & Quercus ilex & KC774594 & KC774594 & KC774547 \\
\hline $\begin{array}{l}\text { Lopadostoma } \\
\text { meridionale }\end{array}$ & LG33 & Quercus coccifera & KC774595 & KC774595 & KC774548 \\
\hline $\begin{array}{l}\text { Lopadostoma cf. } \\
\text { polynesium }\end{array}$ & LAG & $\begin{array}{l}\text { Amelanchier } \\
\text { ovalis }\end{array}$ & KC774600 & KC774600 & KC774553 \\
\hline Lopadostoma pouzarii & CBS 103.96 & $\begin{array}{l}\text { Fraxinus } \\
\text { excelsior }\end{array}$ & KC774601 & KC774601 & KC774554 \\
\hline Lopadostoma pouzarii & MUCL 47149 & $\begin{array}{l}\text { Fraxinus } \\
\text { excelsior }\end{array}$ & KC774602 & KC774602 & NA \\
\hline Lopadostoma quercicola & CBS 133212 & Quercus cerris & KC774603 & KC774603 & KC774555 \\
\hline Lopadostoma quercicola & LG16 & Quercus cerris & KC774606 & KC774606 & KC774556 \\
\hline Lopadostoma quercicola & CBS 134633 & $\begin{array}{l}\text { Quercus } \\
\text { pubescens }\end{array}$ & KC774610 & KC774610 & KC774558 \\
\hline Lopadostoma turgidum & LT & Fagus sylvatica & KC774616 & KC774616 & KC774561 \\
\hline Lopadostoma turgidum & LT1 & Fagus sylvatica & KC774617 & KC774617 & KC774562 \\
\hline Lopadostoma turgidum & CBS 133207 & Fagus sylvatica & KC774618 & KC774618 & KC774563 \\
\hline
\end{tabular}

Results

Diaporthales based on analyses of LSU and ITS sequence data.

Diaporthales is a well-resolved ascomycetous order comprising phytopathogenic, saprobic, and endophytic fungi (Senanayake et al. 2017). Pseudomelanconidaceae was proposed in Diaporthales by Fan et al. (2018), and can be distinguished from the other diaporthalean taxa by conidiogenous cells with apical collarets and discreet annellations, and the inconspicuous hyaline conidial sheath when mature. Pseudomelanconis caryae on Carya cathayensis is the sole species in Pseudomelanconidaceae and causes branch canker of hickory trees in China (Fan et al. 2018).

The taxa in Diaporthales were selected based on Wijayawardene et al. (2017, 2018). The combined LSU and ITS data set consisted of 53 strains with Nakataea oryzae (CBS 243.76) and Pyricularia grisea (Ina168) as outgroup taxa. Alignment comprises 1537 characters after alignment. Of these, 920 characters were constant, 120 variable characters were parsimonyuninformative and 507 characters were parsimony informative. The MP analysis resulted in ten equally most parsimonious trees, with the first tree $(\mathrm{TL}=2609, \mathrm{CI}=0.419, \mathrm{RI}=0.606, \mathrm{RC}=$ 0.254 ) was shown in Fig. 2. The topology of phylogenetic tree obtained from ML was similar with the MP tree. The novel genus appeared in a distinct clade with high bootstrap support (Fig. 2).

Neopseudomelanconis C.M. Tian \& N. Jiang, gen. nov.

MycoBank number: MB 825183; Facesoffungi number: FoF 04969

Etymology - Named after its morphological similarity to Pseudomelanconis.

Type species: Neopseudomelanconis castaneae C.M. Tian \& N. Jiang.

Original description - Sexual morph: Undetermined. Asexual morph - Conidiomata in bark, acervular, immersed in host bark to erumpent. Ectostromatic disc inconspicuous, causing a more or less pustulate bark surface. Central column beneath the disc more or less conical. The marginal part of the central column comprises conidiophores and their basal cell layers. Conidiophores branched 
or unbranched, aseptate, cylindrical, hyaline to pale brown, smooth-walled, sometimes reduced to conidiogenous cells. Conidiogenous cells annellidic, sometimes with apical collarette. Conidia hyaline when immature, becoming brown at maturity, ellipsoid to oblong, 2-celled, multiguttulate, with distinct hyaline sheath, becoming inconspicuous when mature. Conidial wall smooth on the outer surface, with inconspicuous to distinct, sometimes confluent irregular verrucae on the inner surface.

Table 4 Strains of Myrmaecium species used in the molecular analyses in this study, the genes sequenced and GenBank accessions. The new strains from the current study are in red.

\begin{tabular}{|c|c|c|c|c|c|c|c|}
\hline \multirow{2}{*}{ Species } & \multirow{2}{*}{ Strain/Specimen } & \multirow{2}{*}{ Host } & \multirow{2}{*}{ Country } & \multicolumn{4}{|c|}{ GenBank numbers } \\
\hline & & & & ITS & $\mathbf{L S U}$ & TEF & RPB2 \\
\hline Bambusaria bambusae & MFLUCC 12-0851 & Thyrsostachys siamensis & Thailand & KP687812 & KP687812 & KP687982 & KP687890 \\
\hline Bambusaria bambusae & CBS 139763 & Thyrsostachys siamensis & Thailand & KP687813 & KP687813 & KP687983 & KP687891 \\
\hline Myrmaecium fulvopruinatum & CBS 139057 & Fagus sylvatica & Austria & KP687858 & KP687858 & KP688027 & KP687933 \\
\hline Myrmaecium fulvopruinatum & CBS 139058 & Fagus grandifolia & USA & KP687861 & KP687861 & KP688030 & KP687936 \\
\hline Myrmaecium fulvopruinatum & CBS 139059 & Quercus cerris & Austria & KP687863 & KP687863 & KP688032 & KP687938 \\
\hline Myrmaecium fulvopruinatum & VFJ1 & $\begin{array}{l}\text { Unidentified corticated } \\
\text { twigs }\end{array}$ & China & KP687862 & KP687862 & KP688031 & KP687937 \\
\hline Myrmaecium fulvopruinatum & CFCC 52769 & Castanea mollissima & China & MH458908 & MH458902 & MH482828 & MH482835 \\
\hline Myrmaecium fulvopruinatum & CFCC 52770 & Castanea mollissima & China & MH458909 & MH458903 & MH482829 & MH482836 \\
\hline Myrmaecium fulvopruinatum & CFCC 52771 & Castanea mollissima & China & MH458910 & MH458904 & MH482830 & MH482837 \\
\hline Myrmaecium fulvopruinatum & CFCC 52772 & Castanea mollissima & China & MH458911 & MH458905 & MH482831 & MH482838 \\
\hline Myrmaecium fulvopruinatum & CFCC 52773 & Castanea mollissima & China & MH458912 & MH458906 & MH482832 & MH482839 \\
\hline Myrmaecium fulvopruinatum & CFCC 52774 & Castanea mollissima & China & MH458913 & MH458907 & MH482833 & MH482834 \\
\hline Myrmaecium rubricosum & CBS 139067 & Unidentified bark & France & KP687881 & KP687881 & KР688049 & KP687955 \\
\hline Myrmaecium rubricosum & CBS 139068 & Quercus pubescens & Croatia & KP687885 & KP687885 & KP688053 & KP687958 \\
\hline Myrmaecium rubrum & CBS 109505 & Quercus sp. & Italy & GU456324 & GU456324 & GU456260 & GU456344 \\
\hline Valsaria insitiva & CBS 139056 & Acer monspessulanum & Croatia & KP687847 & KP687847 & KP688016 & KP687922 \\
\hline Valsaria insitiva & CBS 139061 & $\begin{array}{l}\text { unidentified corticated } \\
\text { twigs }\end{array}$ & China & KP687866 & KP687866 & KP688035 & KP687941 \\
\hline
\end{tabular}


Table 4 Continued.

\begin{tabular}{|c|c|c|c|c|c|c|c|}
\hline \multirow[b]{2}{*}{ Species } & \multirow[b]{2}{*}{ Strain/Specimen } & \multirow[b]{2}{*}{ Host } & \multirow[b]{2}{*}{ Country } & \multicolumn{4}{|c|}{ GenBank numbers } \\
\hline & & & & ITS & LSU & TEF & RPB2 \\
\hline Valsaria lopadostomoides & CBS 139062 & Quercus ilex & Greece & KP687868 & KP687868 & KP688037 & KP687943 \\
\hline Valsaria neotropica & CBS 139064 & $\begin{array}{l}\text { Unidentified corticated } \\
\text { twig }\end{array}$ & France & KP687874 & KP687874 & KP688042 & KP687948 \\
\hline Valsaria robiniae & CBS 121890 & Hippocrepis emerus & Slovenia & KP687851 & KP687851 & KР688020 & KP687926 \\
\hline Valsaria robiniae & CBS 128015 & Caragana arborescens & Austria & KP687853 & KP687853 & KP688022 & KP687928 \\
\hline Valsaria rudis & CBS 139065 & Quercus macrolepis & Greece & KP687878 & KP687878 & KP688046 & KP687952 \\
\hline Valsaria rudis & CBS 139066 & Quercus pubescens & Austria & KP687879 & KР687879 & КР688047 & KP687953 \\
\hline Valsaria spartii & CBS 121714 & Ceratonia siliqua & Greece & KP687848 & KP687848 & KP688017 & KP687923 \\
\hline Valsaria spartii & CBS 125584 & Cytisus scoparius & Italy & EU040213 & EU040213 & KP688023 & KP687929 \\
\hline
\end{tabular}

Neopseudomelanconis castaneae C.M. Tian \& N. Jiang, sp. nov.

MycoBank number: MB 825198; Facesoffungi number: FoF 04970

Etymology - Named after the host genus from which it was isolated, Castanea.

Saprobic on Castanea mollissima Blume in terrestrial habitats. Sexual morph - Undetermined. Asexual morph - Conidiomata, 0.5-1.5 mm wide $(\overline{\mathrm{x}}=0.8, \mathrm{SD} \pm 0.2 \mathrm{~mm}, \mathrm{n}=20), 0.2-0.5 \mathrm{~mm}$ high $(\overline{\mathrm{x}}=0.3, \mathrm{SD} \pm 0.15 \mathrm{~mm}, \mathrm{n}=20)$, acervular, immersed in host bark to erumpent, covered by blackish conidial masses at maturity. Central column beneath the disc more or less conical. Conidiophores unbranched or branched at base, aseptate, cylindrical, hyaline to pale brown, smooth-walled, $10-35 \times 3.5-6 \mu \mathrm{m}(\overline{\mathrm{x}}=25, \mathrm{SD} \pm 5.5 \mu \mathrm{m} \times \overline{\mathrm{x}}=4.5$, $\mathrm{SD} \pm 1.5 \mu \mathrm{m}, \mathrm{n}=20)$. Conidiogenous cells enteroblastic, annellidic, with distinct annellations and collarettes. Conidia hyaline when immature, becoming greyish sepia to olivaceous, ellipsoid to oblong, multiguttulate, straight to slightly curved, 2-celled, $18-21.5 \times 4.8-7 \mu \mathrm{m}(\overline{\mathrm{x}}=19.5, \mathrm{SD} \pm 1.2 \mu \mathrm{m} \times \overline{\mathrm{x}}=5.9, \mathrm{SD} \pm 0.6 \mu \mathrm{m}, \mathrm{n}=50), 1 / \mathrm{w}=(2.7-$ )2.9-3.7(-4.2), with distinct hyaline sheath, $0.5-1 \mu \mathrm{m}$ diam., becoming inconspicuous when mature. Conidial wall smooth on the outer surface.

Culture characteristics - On PDA at $25{ }^{\circ} \mathrm{C}$, cultures are initially white, becoming grey olivaceous. The colonies are flat, with irregular margins; texture initially uniform, becoming compact in centre after 1 month.

Known distribution - Dead corticated branches of Castanea mollissima.

Material examined - CHINA, Shaanxi Province, Ankang City, chestnut plantation, 33³9'27.34"N, 10907'15.23"E, 2504 m asl, on branches of Castanea mollissima Blume (Fagaceae), N. Jiang, 8 July 2017 (BJFC-S1444, holotype), ex-holotype CFCC 52787. Shaanxi Province, Ankang City, chestnut plantation, $32^{\circ} 13^{\prime} 43.51^{\prime \prime} \mathrm{N}, 1^{\circ} 9^{\circ} 00^{\prime} 44.24^{\prime \prime} \mathrm{E}, 1810 \mathrm{~m}$ asl, on branches of Castanea mollissima, N. Jiang, 3 July 2017 (BJFC-S1445, paratype), living culture CFCC 52788. 
Notes - Two isolates of Neopseudomelanconis castaneae cluster in a well-supported clade (MP/ML = 86/100) sister to Pseudomelanconis caryae (Fig. 2). Neopseudomelanconis castaneae and Pseudomelanconis caryae are united in the family Pseudomelanconidaceae by conidiogenous cells with apical collarets and discreet annellations, and the hyaline conidial sheath. However, Neopseudomelanconis is different from Pseudomelanconis in having branched conidiophores and 2-celled conidia. Additionally, Neopseudomelanconis castaneae and Pseudomelanconis caryae are the only two species in Pseudomelanconidaceae, they can be distinguished in conidial dimension $(18-21.5 \times 4.8-7 \mu \mathrm{m}$ in Neopseudomelanconis castaneae vs. $13-15 \times 4-5 \mu \mathrm{m}$ in Pseudomelanconis caryae), and ITS and LSU loci (35/512 in ITS and 11/557 in LSU).

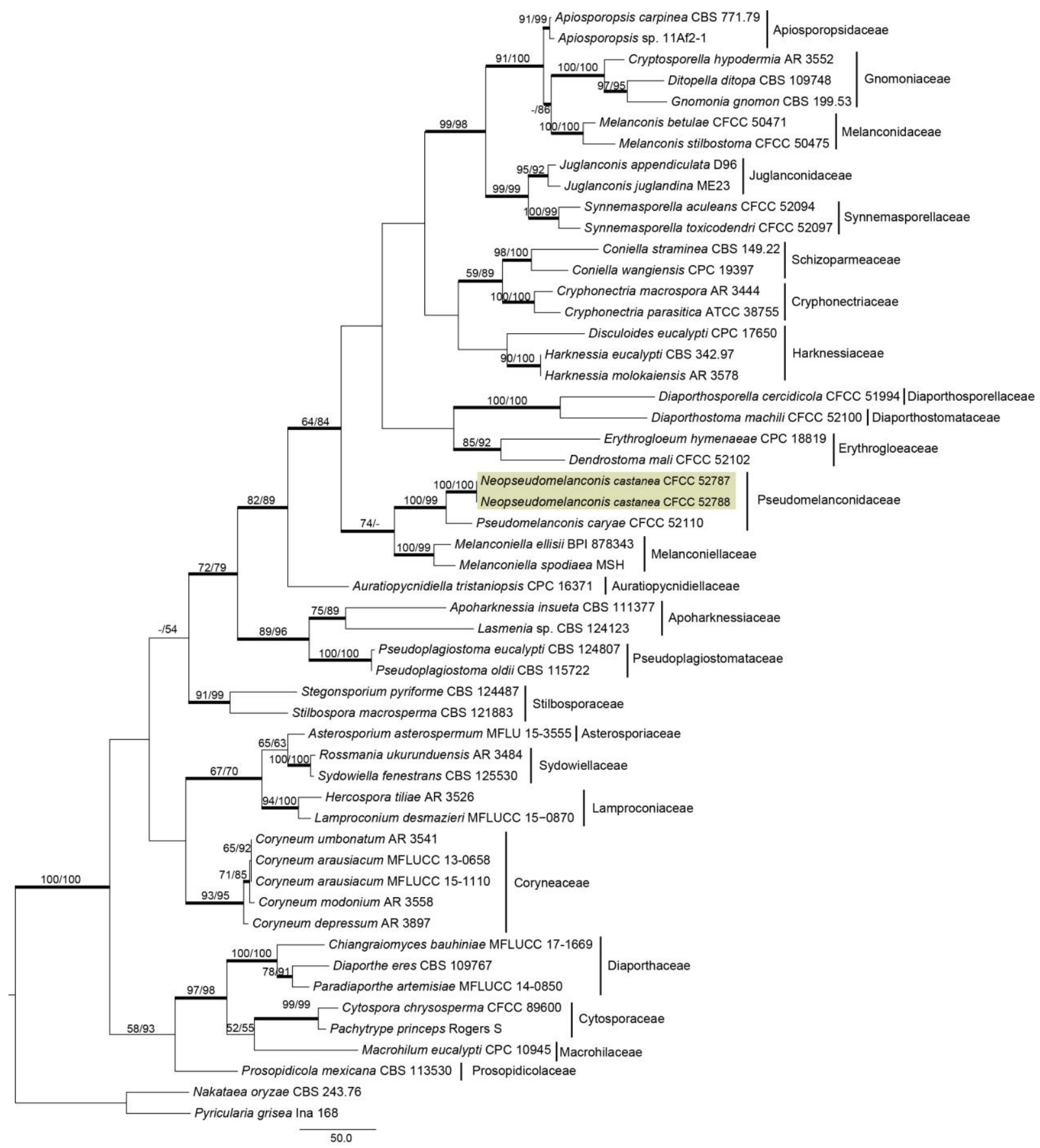

Figure 2 - Phylogram of Diaporthales based on combined LSU and ITS genes. MP and ML bootstrap support values above $50 \%$ are shown at the first and second position. Thickened branches represent posterior probabilities above 0.90 from Bayesian inference. Scale bar $=50$ nucleotide substitutions. The new sequences resulting from the current study are highlighted in yellow. 

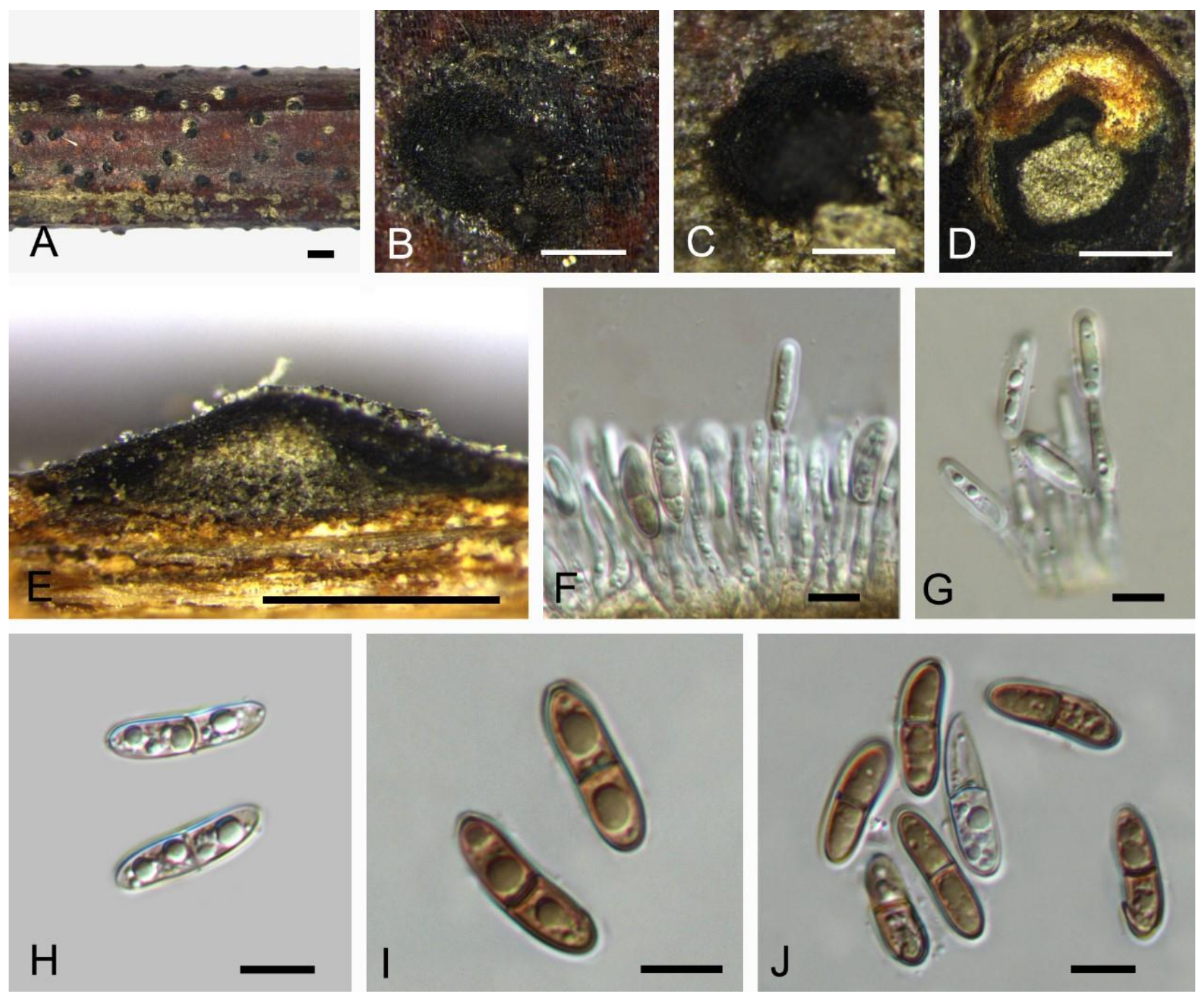

Figure 3 - Morphology of Neopseudomelanconis castaneae from Castanea mollissima (BJFCS1444, holotype). A-C Habit of conidiomata on a stem. D Transverse sections through conidiomata. E Longitudinal sections through conidiomata. F, G Developing conidia attached to conidiogenous cells. $\mathrm{H}$ Immature conidia. I, J Mature conidia. Scale bars: A $=1 \mathrm{~mm}, \mathrm{~B}-\mathrm{E}=0.5$ $\mathrm{mm}, \mathrm{F}-\mathrm{J}=10 \mu \mathrm{m}$.

\section{Aplosporella based on analyses of ITS and TEF1- $\alpha$ sequence data.}

Aplosporella (Aplosporellaceae, Botryosphaeriales) can be recognized by multiloculate conidiomata with a single ostiole, and brown, aseptate conidia (Du et al. 2017). The combined ITS and TEF1- $\alpha$ data set consists of 19 strains with Saccharata proteae (CBS 115206) as outgroup taxon. Alignment comprises 739 characters. Of these, 429 characters were constant, 229 variable characters were parsimony-uninformative and 81 characters were parsimony informative. The MP analysis resulted in 4 equally most parsimonious trees, with the first tree $(\mathrm{TL}=383, \mathrm{CI}=0.903$, RI $=0.805, \mathrm{RC}=0.727$ ) was shown in Fig. 4 . The topology of phylogenetic tree obtained from ML was similar with the MP tree.

Aplosporella javeedii Jami, Gryzenh., Slippers \& M.J. Wingf., Fungal Biology 118: 174 (2014).

Fig. 5

Saprobic on dead and corticated branches of various hosts in terrestrial habitats. Sexual morph - Undetermined. Asexual morph - Conidiomata immersed in bark, erumpent from bark surface, separate, discoid, dark-brown to black, multi-locular, $0.5-1.5 \mathrm{~mm}$ diam. $(\overline{\mathrm{x}}=0.8, \mathrm{SD} \pm$ $0.25 \mathrm{~mm}, \mathrm{n}=20$ ), wall consisting of 4-10 layers, thick outer layers composed of dark-brown textura angularis, becoming hyaline and thin-walled towards the inner region. Disc brown to black, 
circular to ovoid, $0.42-0.76 \mathrm{~mm}(\overline{\mathrm{x}}=0.55, \mathrm{SD} \pm 0.03 \mathrm{~mm}, \mathrm{n}=20)$, with $1-10$ ostioles per disc. Ostioles multiple, conspicuous, level with the disc surface, sometimes covered below disc by lighter entostroma, $25.1-65.8 \mu \mathrm{m}$ in diam. $(\overline{\mathrm{x}}=50.5, \mathrm{SD} \pm 4.5 \mu \mathrm{m}, \mathrm{n}=20)$. Locules multiple, irregularly arranged, subdivided frequently by invaginations with common walls, $0.80-1.52 \mathrm{~mm}$ in diam. $(\overline{\mathrm{x}}=1.2, \mathrm{SD} \pm 0.15 \mathrm{~mm}, \mathrm{n}=20)$, Paraphyses hyaline, smooth-walled, 25.6-74.2 $\mu \mathrm{m}$ long $(\overline{\mathrm{x}}$ $=45.5, \mathrm{SD} \pm 15 \mu \mathrm{m}, \mathrm{n}=20), 3.2-5.5 \mu \mathrm{m}$ wide $(\overline{\mathrm{x}}=4.1, \mathrm{SD} \pm 0.5 \mu \mathrm{m}, \mathrm{n}=20)$. Conidiogenous cells formed from the cells lining the inner walls of the pycnidia, holoblastic, determinate, simple, ellipsoidal, and slightly tapered towards the apex, hyaline. Conidia aseptate, ellipsoid to oblong, smooth, ends rounded, initial hyaline, becoming brown when mature, 18.1-24.8 $\times 7.5-11.7 \mu \mathrm{m}(\overline{\mathrm{x}}$ $=21.3, \mathrm{SD} \pm 2.2 \mu \mathrm{m} \times \overline{\mathrm{x}}=10.4, \mathrm{SD} \pm 0.8 \mu \mathrm{m}, \mathrm{n}=50), 1 / \mathrm{w}=(2-) 2.1-2.2(-2.2)$.

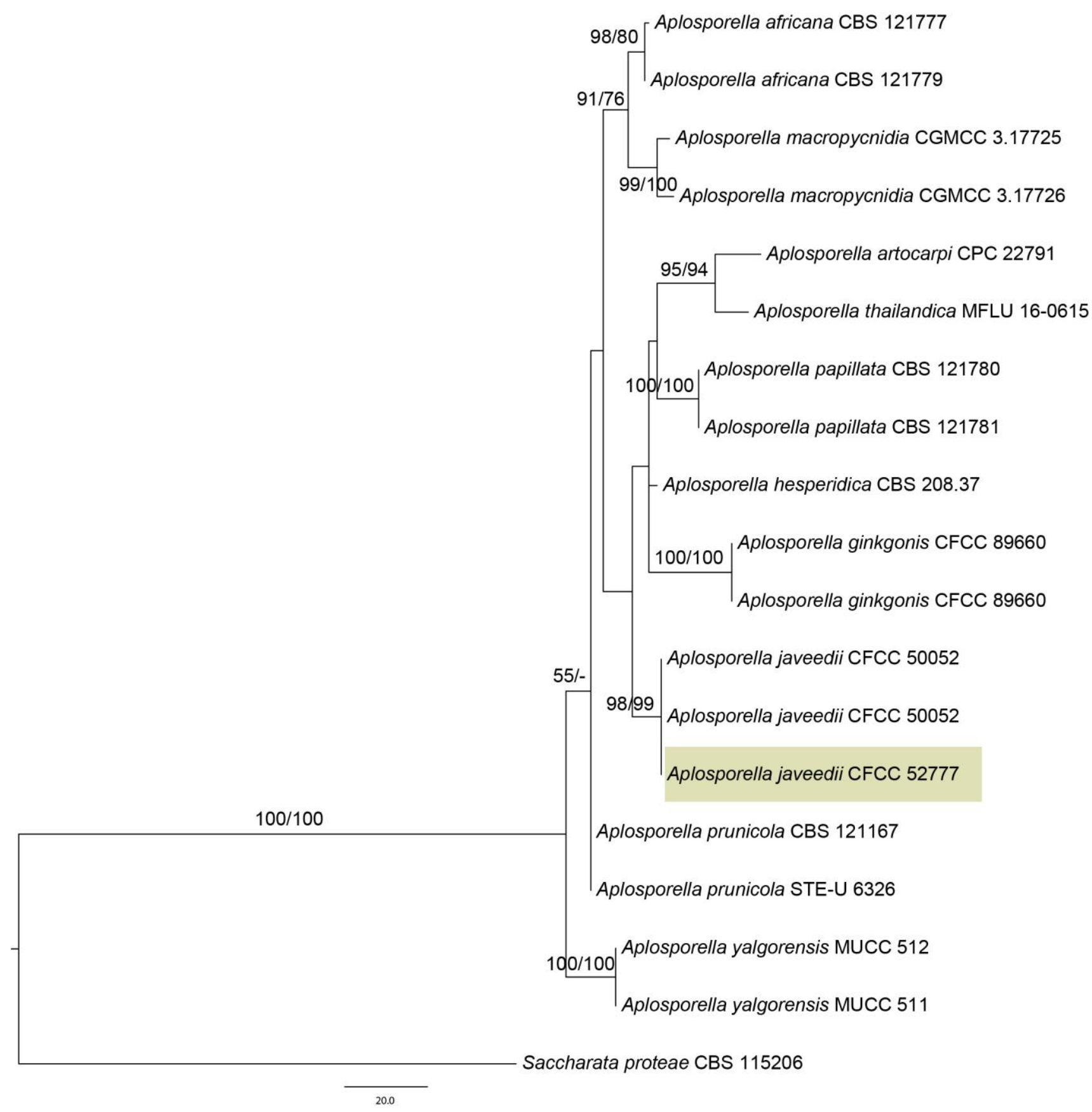

Figure 4 - Phylogram of Aplosporella based on combined ITS and TEF1- $\alpha$ genes. MP and ML bootstrap support values above $50 \%$ are shown at the first and second position. Scale bar $=20$ nucleotide substitutions. The new sequence resulting from the current study is highlighted in yellow. 

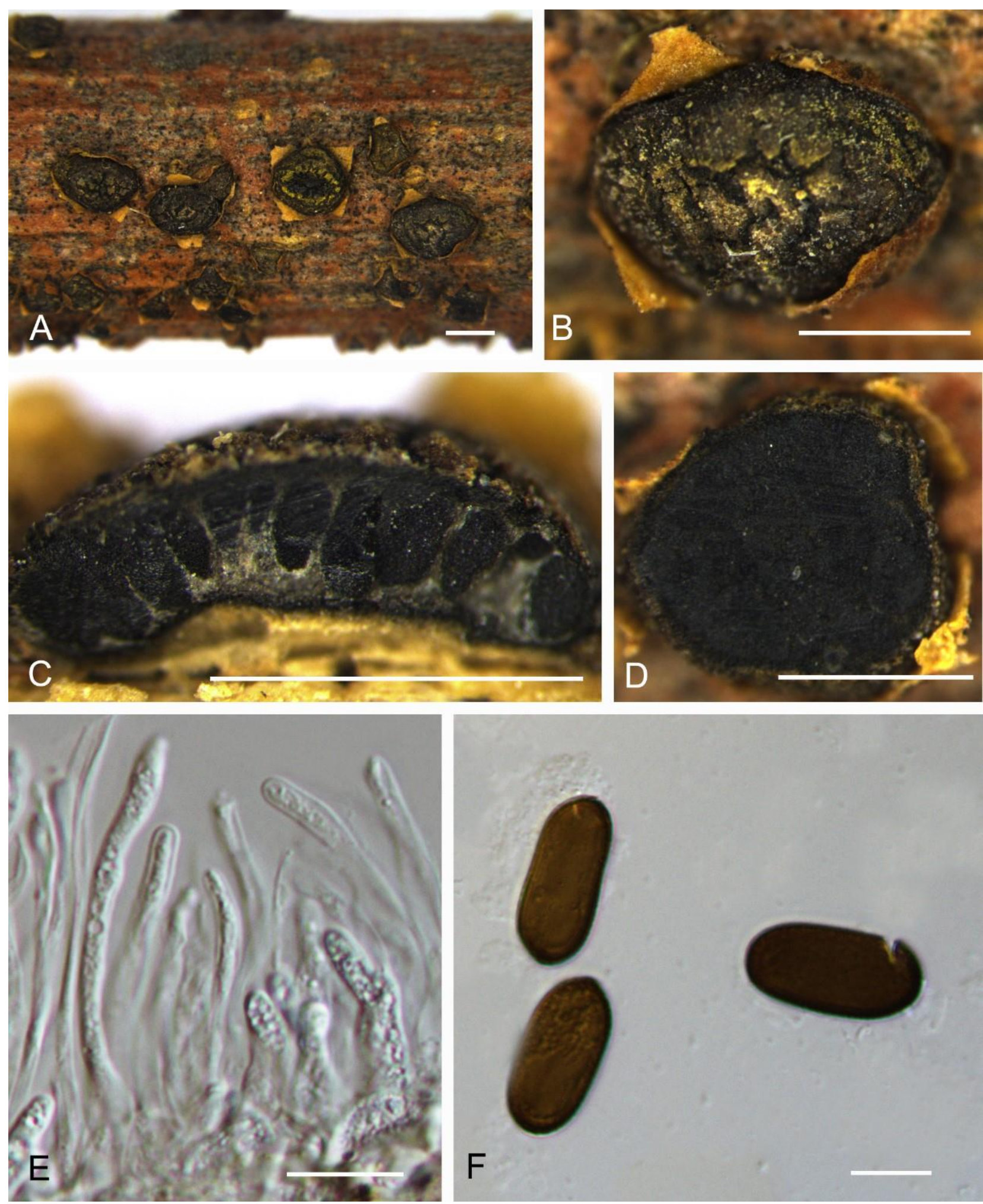

Figure 5 -Aplosporella javeedii from Castanea mollissima (BJFC-S1446). A, B Habit of conidiomata on a stem. C Longitudinal sections through conidiomata. D Transverse sections through conidiomata. E Conidiogenous cells and paraphyses. F conidia. Scale bars: A-D = $1 \mathrm{~mm}$, $\mathrm{E}, \mathrm{F}=10 \mu \mathrm{m}$.

Culture characteristics - On MEA at $25{ }^{\circ} \mathrm{C}$, white at first, then grey-olivaceous, similar to reverse side after 8 days. Colony uniform with appressed aerial mycelium. After 3 weeks, conidiomata solitary, globose, unilocular, immersed to semi-immersed on MEA.

Known distribution - Dead corticated branches of Albizia julibrissin Durazz. (Leguminosae) 
(BJFC-S867), Broussonetia papyrifera L'Hér. ex Vent. (Moraceae) (BJFC-S884), Castanea mollissima Blume (Fagaceae) (BJFC-S1446), Gleditsia sinensis Lam. (Leguminosae) (BJFCS1022), Juniperus chinensis L. (Cupressaceae) (BJFC-S1044), and Styphnolobium japonicum L. (Leguminosae) (BJFC-S1042) in China and from Celtis africana (Ulmaceae) (CMW 38166) and Searsia lancea (Anacardiaceae) (CMW 38168) in South Africa.

Specimen examined - CHINA, Hebei Province, Chengde City, 40³7'17.15"N, $118^{\circ} 28^{\prime} 21.54^{\prime \prime} \mathrm{E}, 325 \mathrm{~m}$ asl, asexual morphs on corticated branches of Castanea mollissima, $\mathrm{N}$. Jiang, 13 October 2017 (BJFC-S1446), living culture CFCC 52777.

Notes - The specimen of Aplosporella collected from chestnut branches is identified as Aplosporella javeedii based on morphological observations and multi-gene phylogenetic analyses. Morphologically, it is characterized by solitary globose unilocular conidiomata on MEA and erumpent discoid conidiomata, multiple ostioles in branches with ellipsoid to oblong aseptate conidia averaging $21.3 \times 10.4 \mu \mathrm{m}$, which matches the type (CBS 133954) perfectly (Jami et al. 2014).

Coryneaceae is characterized by having black perithecia, often immersed in wood, asci that deliquesce at maturity, and an asexual morph with transversely distoseptate brown conidia (Senanayake et al. 2017). Coryneum is the sole genus in the family Coryneaceae of Diaporthales, and asexual morph is very common in nature and sometimes the sexual morph is closely associated with it (Senanayake et al. 2017). Coryneum species are generally considered highly host specific, especially occurring on hardwood trees such as those in the Betulaceae and Fagaceae (Sutton 1980).

Coryneum umbonatum Nees, Syst. Pilze (Würzburg) 34 (1816).

Fig. 6

Pathogenic on Castanea mollissima branches. Sexual morph - Undetermined. Asexual morph - Conidiomata acervular, $0.2-0.8 \mathrm{~mm}$ wide $(\overline{\mathrm{x}}=0.5, \mathrm{SD} \pm 0.25 \mathrm{~mm}, \mathrm{n}=20), 0.2-0.7 \mathrm{~mm}$ high $(\overline{\mathrm{x}}=0.4, \mathrm{SD} \pm 0.2 \mathrm{~mm}, \mathrm{n}=20)$, solitary, erumpent through outer periderm layers of host, scattered, surface tissues above slightly domed. Conidiophores $15-30 \mu \mathrm{m}$ long $(\overline{\mathrm{x}}=24, \mathrm{SD} \pm 4.5$ $\mu \mathrm{m}, \mathrm{n}=20), 3-7 \mu \mathrm{m}$ wide $(\overline{\mathrm{x}}=4.5, \mathrm{SD} \pm 1.5 \mu \mathrm{m}, \mathrm{n}=20)$, branched at base, cylindrical, septate, hyaline at apex, pale brown at base. Conidiogenous cells holoblastic, annellidic, integrated, indeterminate, cylindrical, expanding towards apices, pale brown, smooth, with $0-1$ percurrent proliferations. Conidia 42.2-82.5 $\times 9.6-15.7 \mu \mathrm{m}(\overline{\mathrm{x}}=62.5, \mathrm{SD} \pm 6.5 \mu \mathrm{m} \times \overline{\mathrm{x}}=13.6, \mathrm{SD} \pm 1.1 \mu \mathrm{m}$, $\mathrm{n}=50), 1 / \mathrm{w}=(4.2-) 4.4-4.7(-5.1)$, variable in shape, curved, broadly fusiform to fusiform cylindrical or clavate, dark brown, smooth-walled, 5-7-distoseptate, apical cell with a hyaline tip, truncate and black at base.

Known distribution - on stems of Betula sp. (BPI 871006A) in the USA, on twigs of Quercus petraea Libelb (Fagaceae) (IMI 362542) in the UK and on branches of Castanea mollissima in China.

Specimen examined - CHINA, Hebei Province, Qinhuangdao City, 40²2'51.22"N, $119^{\circ} 11^{\prime} 51.15^{\prime E}$, $235 \mathrm{~m}$ asl, asexual morphs on branches of Castanea mollissima, collected by N. Jiang, 16 October 2017 (BJFC-S1449).

Notes - Coryneum umbonatum could cause cankers, dieback in shoots and twigs (Sutton 1980). In the present study, a Coryneum specimen collected from dead chestnut branches is identified as Coryneum umbonatum exactly matched conidial dimensions (Sutton 1980). Coryenum modonium was reported on Castanea species, but conidial size can differ the two species (42.2$82.5 \times 9.6-15.7 \mu \mathrm{m}$ in Coryneum umbonatum vs. 50-71 $\times 14-19 \mu \mathrm{m}$ in C. modonium) (Sutton 1975, Teng 1996).

\section{Lopadostoma based on analyses of LSU, ITS and RPB2 sequence data.}

Lopadostoma (Lopadostomataceae, Xylariales) was reported as a saprobiotic genus with pustular pseudostroma development in bark of angiospermous trees (Jaklitsch et al. 2014). Species of Lopadostoma are generally not particularly common, but show a marked seasonal occurrence. $L$. americanum, L. dryophilum, L. insulare, L. meridionale and L. quercicola, were only collected 
from Quercus trees, and these five spices were close in the phylogram (Jaklitsch et al. 2014). However, none was recorded from China.

The combined LSU, ITS and RPB2 data set consists of 30 strains with Lopadostoma cf. polynesium (LAG) as outgroup taxon. Alignment comprises 2750 characters after alignment. Of these, 1917 characters were constant, 255 variable characters were parsimony-uninformative and 578 characters were parsimony informative. The MP analysis resulted in one parsimonious tree (TL $=1464, \mathrm{CI}=0.750, \mathrm{RI}=0.861, \mathrm{RC}=0.646$ ), which was shown in Fig. 7 . The topology of phylogenetic tree obtained from ML was similar with the MP tree.

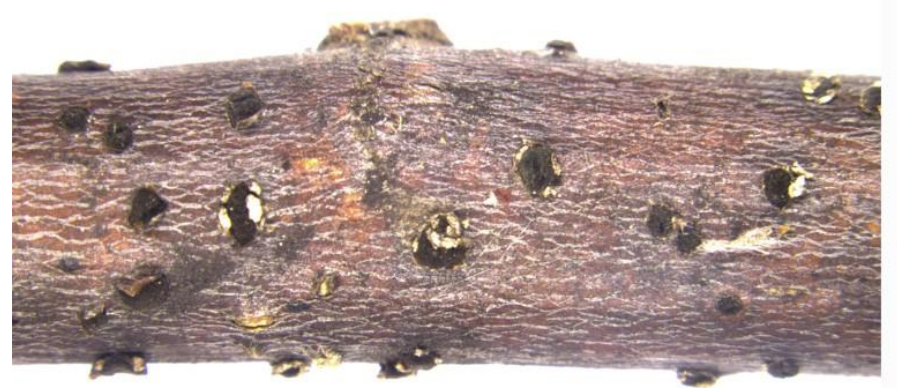

A
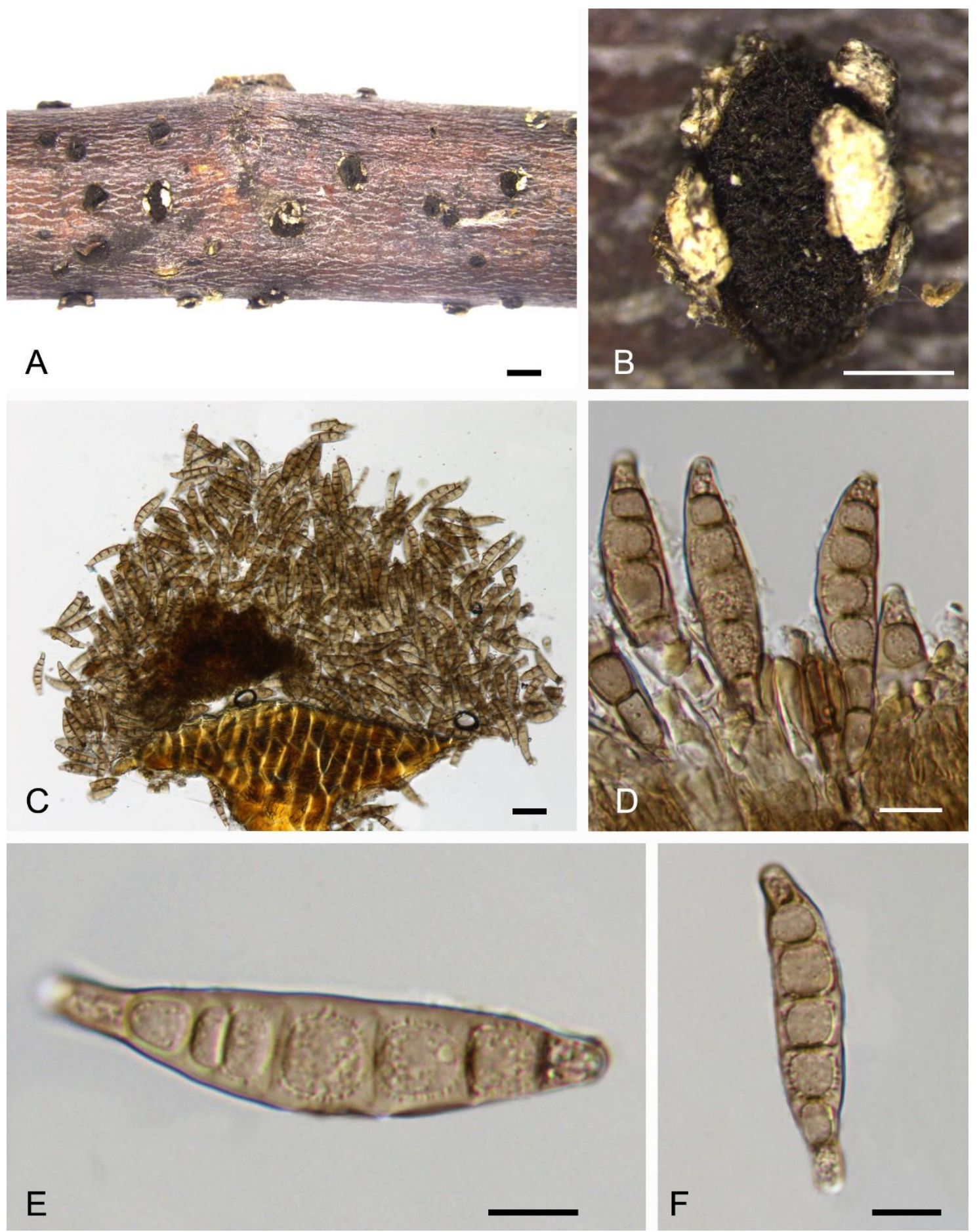

Figure 6 - Morphology of Coryneum umbonatum from Castanea mollissima (BJFC-S1449). A-B Habit of conidiomata on a stem. D Conidiogenous cells. C, E-F conidia. Scale bars: A = 1 $\mathrm{mm}, \mathrm{B}=0.5 \mathrm{~mm}, \mathrm{C}=30 \mu \mathrm{m}, \mathrm{E}-\mathrm{F}=10 \mu \mathrm{m}$. 


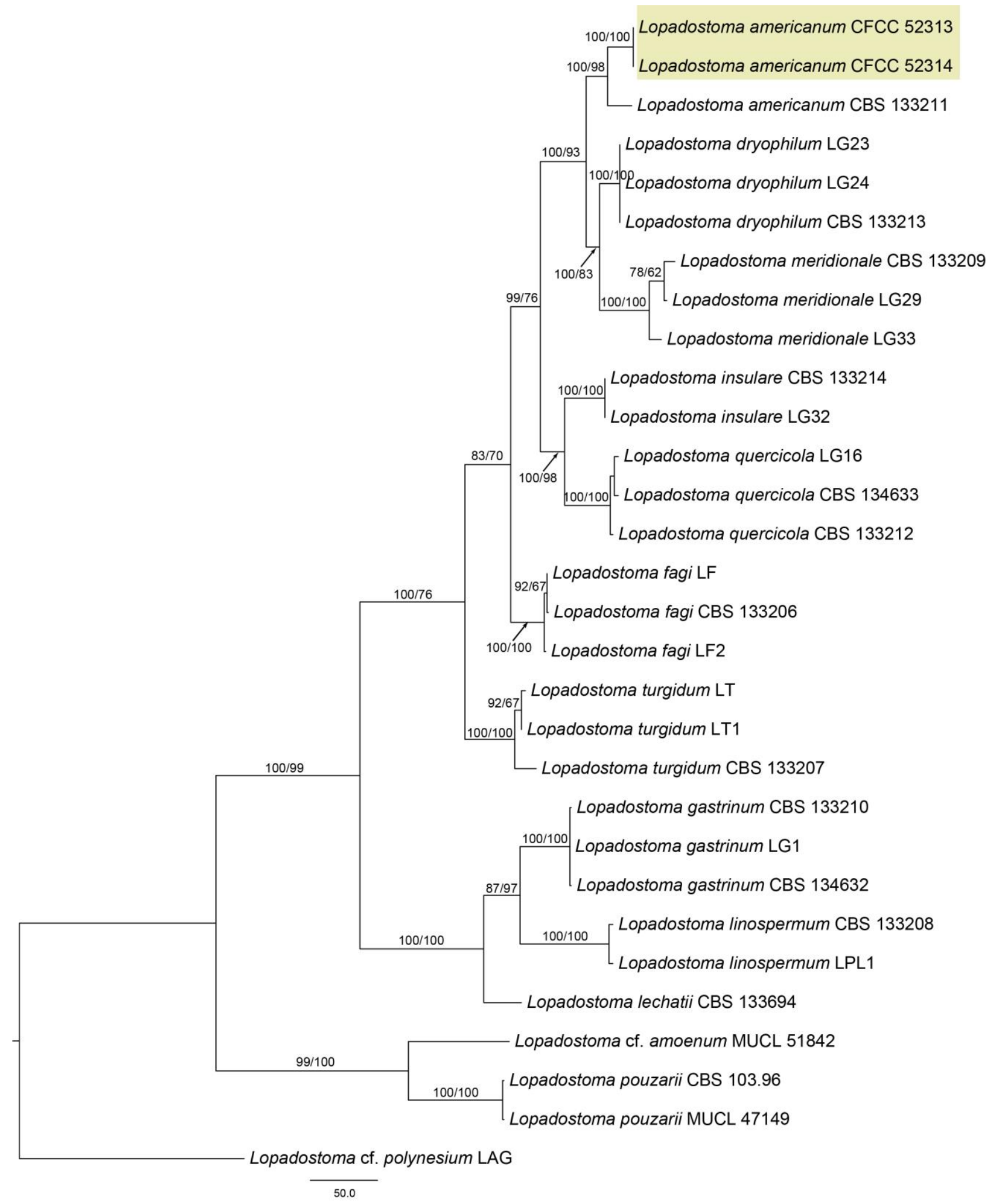

Figure 7 - Phylogram of Lopadostoma based on combined LSU, ITS and RPB2 genes. MP and ML bootstrap support values above $50 \%$ are shown at the first and second position. Scale bar $=20$ nucleotide substitutions. The new sequences resulting from the current study are highlighted in yellow.

Lopadostoma americanum Jaklitsch, J. Fourn., J.D. Rogers \& Voglmayr, Persoonia 32: 58 (2014). Fig. 8

Saprobic on Castanea mollissima branches. Sexual morph - Ectostromatic discs convex, circular or ellipsoid in outline, $0.8-1 \mathrm{~mm}$ in their greatest dimension, projecting to $0.5 \mathrm{~mm}$ above 
the host surface, roughened, carbonaceous, shiny in places; ostioles opening separately in the disc, inconspicuous, umbilicate. Stromata for the most part immersed in bark, pustulate-erumpent, separate, scattered, subglobose, $1.5-2.5 \mathrm{~mm}$ diam. $(\overline{\mathrm{x}}=2, \mathrm{SD} \pm 0.5 \mathrm{~mm}, \mathrm{n}=20)$, delimited from surrounding bark by a black carbonized line; the latter 80-120 $\mu \mathrm{m}$ thick, irregular in outline, reaching the wood surface and spreading over it between adjacent stromata. Tissue between ostiolar necks dark yellow-brown, grey to black, woody; tissue around and beneath the perithecia composed of brownish, scarcely altered bark tissue, in places mixed with whitish mycelium. Perithecia 6-10 per stroma, arranged in valsoid configuration, monostichous in smaller stromata, polystichous in larger ones, subglobose to ovoid, often laterally com-pressed, $0.2-0.7 \mathrm{~mm}$ diam. $(\overline{\mathrm{x}}=0.5, \mathrm{SD} \pm$ $0.25 \mathrm{~mm}, \mathrm{n}=20$ ), with long ostiolar necks converging toward the ectostromatic disc, $0.8-1.2 \mathrm{~mm}$. Asci cylindrical, with 8 uniseriate ascospores, $52-88 \times 5.1-8.4 \mu \mathrm{m},(\overline{\mathrm{x}}=70, \mathrm{SD} \pm 9.5 \mu \mathrm{m} \times \overline{\mathrm{x}}=7.1$, $\mathrm{SD} \pm 0.5 \mu \mathrm{m}, \mathrm{n}=50$ ), sometimes fusiform due to partly overlapping biseriate or oblique ascospores, flat amyloid apical ring, stipe up to nearly as long as the spore part. Ascospores oblong to narrowly ellipsoid, $8-12 \times 3-4.2 \mu \mathrm{m},(\overline{\mathrm{x}}=10, \mathrm{SD} \pm 0.5 \mu \mathrm{m} \times \overline{\mathrm{x}}=3.6, \mathrm{SD} \pm 0.3 \mu \mathrm{m}, \mathrm{n}=50), 1 / \mathrm{w}$ $=(2.1-) 2.5-2.9(-3.3)$, dark to blackish brown, smooth, with 2 guttules when young, with commonly visible, straight, circumferential germ slit. Asexual morph - Undetermined.

Culture characteristics - On PDA at $25^{\circ} \mathrm{C}$, colonies growing slowly, with uneven white mat, colourless, white to yellowish margin, and yellowish reverse, reaching $70 \mathrm{~mm}$ within 50 days, forming large thick yellowish pustules without conidial drops.

Known distribution - On Quercus spp. (CBS 133211) in North America and dead corticated branches of Castanea mollissima in China.

Specimen examined - CHINA, Shandong Province, Rizhao City, 11946'23.18"N, $35^{\circ} 42^{\prime 28.12 " E, ~} 452 \mathrm{~m}$ asl, on branches of Castanea mollissima, collected by N. Jiang, 23 April 2017 (BJFC-S1384, living culture CFCC 52313); Rizhao City, 11946'23.18"N, 3542'28.12"E, $452 \mathrm{~m}$ asl, on branches of Castanea mollissima, collected by N. Jiang, 23 April 2017 (BJFCS1385), living culture CFCC 52314.

Notes - Two isolates of Lopadostoma americanum (CFCC 52313 and CFCC52314) cluster in a well-supported clade (MP/ML=100/100) sister to type strain of Lopadostoma americanum (CBS 133211). In addition, we compared morphological characteristics of Lopadostoma americanum between specimens collected from Castanea mollissima in China and type material recorded by Jaklitsch et al. (2014). Sexual morphs including pyrenocarp and ascospore characteristics and dimensions matched exactly. Hence we do not propose CFCC 52313 and CFCC52314 as a novel species.

\section{Myrmaecium based on analyses of LSU, ITS, TEF1- $\alpha$ and RPB2 sequence data.}

Myrmaecium (Valsariaceae, Valsariales) is a genus traditionally treated as a synonym of Valsaria, but resolved by Jaklitsch et al. (2015) to comprise M. fulvopruinatum, M. rubricosum and M. rubrum. The combined LSU, ITS, TEF1- $\alpha$, and RPB2 data set consists of 25 strains with Bambusaria bambusae (MFLUCC 12-0851) and Bambusaria bambusae (CBS 139763) as outgroup taxa. Alignment comprises 3898 characters after alignment. Of these, 2714 characters were constant, 110 variable characters were parsimony-uninformative and 1074 characters were parsimony informative. The MP analysis resulted in one parsimonious tree $(\mathrm{TL}=2133, \mathrm{CI}=0.755$, $\mathrm{RI}=0.920, \mathrm{RC}=0.245$ ), which was shown in Fig. 9. The topology of phylogenetic tree obtained from ML was similar with the MP tree.

\section{Myrmaecium fulvopruinatum (Berk.) Jaklitsch \& Voglmayr, Fungal Diversity 73: 190 (2015).}

Pathogenic or saprobic on Castanea mollissima branches. Sexual morph - Stromata eustromatic, immersed to erumpent, causing small bumps in bark, subpulvinate, labiate or ring-like, often longish, separate, $1-3 \times 0.5-2 \mathrm{~mm}(\overline{\mathrm{x}}=2, \mathrm{SD} \pm 0.6 \mathrm{~mm} \times \overline{\mathrm{x}}=1.2, \mathrm{SD} \pm 0.3 \mathrm{~mm}, \mathrm{n}=20)$, aggregated in linear rows. Ascomata $0.1-0.4 \mathrm{~mm}$ diam. $(\overline{\mathrm{x}}=0.25, \mathrm{SD} \pm 0.1 \mathrm{~mm}, \mathrm{n}=20), 0.1-0.4$ $\mathrm{mm}$ high $(\overline{\mathrm{x}}=0.25, \mathrm{SD} \pm 0.15 \mathrm{~mm}, \mathrm{n}=20)$, arranged monostichously in valsoid groups of $5-10$ at 
near basal position or in the stroma middle, less commonly in diatrypoid configuration just below the stroma surface, flask-shaped to subglobose; peridium 15-25 $\mu \mathrm{m}$ thick, composed of a thin outer layer of flattened dark brown cells and an inner layer of flattened hyaline cells.
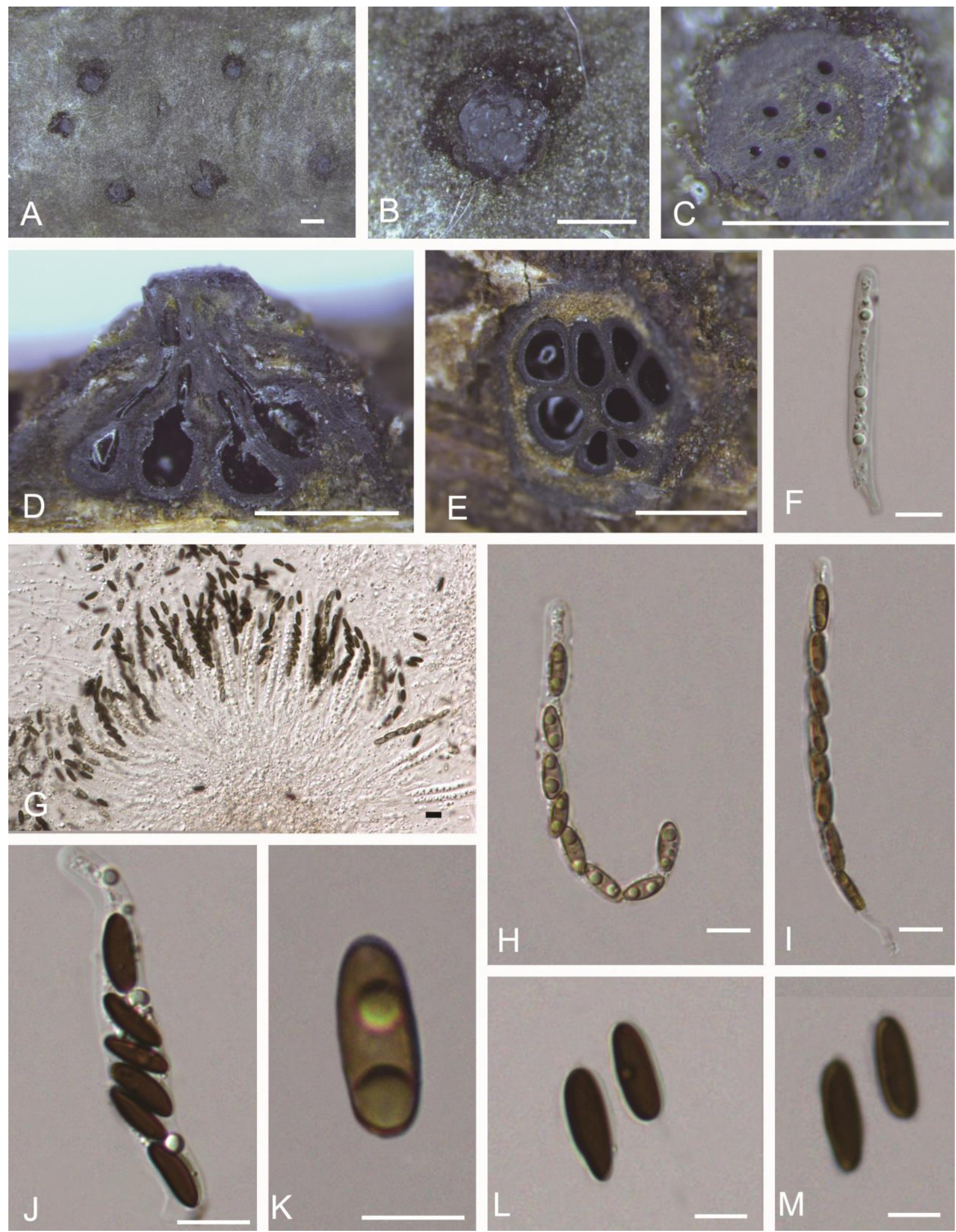

Figure 8 - Morphology of Lopadostoma americanum from Castanea mollissima (BJFC-S1384). A-B Habit of ascomata on a stem. C, E Transverse sections through ascomata. D Longitudinal sections through ascomata. F-J Asci. K: Immature ascospore. L-M Mature ascospores. Scale bars: $\mathrm{A}-\mathrm{E}=1 \mathrm{~mm}, \mathrm{~F}-\mathrm{M}=10 \mu \mathrm{m}$. 


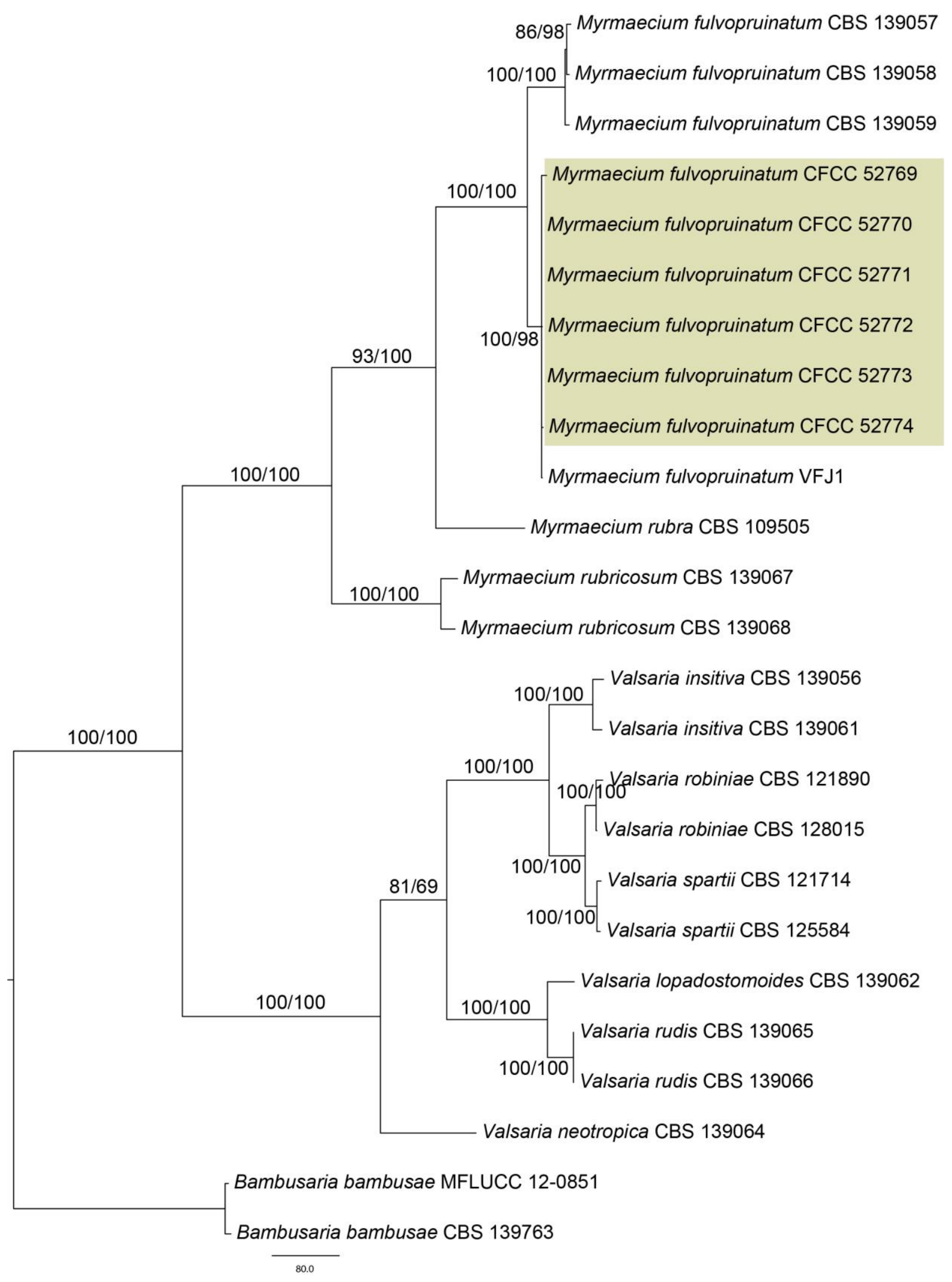

Figure 9 - Phylogram of Myrmaecium based on combined LSU, ITS, TEF1- $\alpha$ and RPB2 genes. MP and ML bootstrap support values above $50 \%$ are shown at the first and second position. Scale bar $=80$ nucleotide substitutions. The new sequences resulting from the current study are highlighted in yellow.

Ostiolar necks converging into a common neck, less commonly separate, $200-1100 \mu \mathrm{m}$ long, $(\overline{\mathrm{x}}=$ 500, SD $\pm 150 \mu \mathrm{m}, \mathrm{n}=20), 90-300 \mu \mathrm{m}$ diam. $(\overline{\mathrm{x}}=200, \mathrm{SD} \pm 50 \mu \mathrm{m}, \mathrm{n}=20)$, black, smooth; apices 
at the surface usually with distinct circular outline, flush with the stroma surface or projecting to $120 \mu \mathrm{m}$; periphysate. Paraphyses filiform, unbranched, apically free, up to $150 \mu \mathrm{m}$ long, $1-5 \mu \mathrm{m}$ wide, attenuated upwards, slightly enlarged at the tip. Asci $88-142 \times 10.8-19.5 \mu \mathrm{m}(\overline{\mathrm{x}}=118, \mathrm{SD} \pm$ $6 \mu \mathrm{m} \times \overline{\mathrm{x}}=14.5, \mathrm{SD} \pm 2.1 \mu \mathrm{m}, \mathrm{n}=20$ ), numerous, cylindrical to subclavate, containing 8 uniseriate ascospores. Ascospores 14.3-19 $\times 7.1-8.6 \mu \mathrm{m}(\overline{\mathrm{x}}=16.5, \mathrm{SD} \pm 1.8 \mu \mathrm{m} \times \overline{\mathrm{x}}=7.8, \mathrm{SD} \pm 0.5 \mu \mathrm{m}, \mathrm{n}=$ $50), 1 / \mathrm{w}=(1.9-) 2-2.4(-2.5)$, ellipsoid, 2-celled, dark brown to black, plump or attenuated towards apices with 1 large drop per cell and densely reticulate surface ornamentation. Asexual morph Immature stromata often with a dark and gelatinous flat central surface, containing conidiomata as irregular, labyrinthine locules at ostiolar levels above immature ascomata; interior of locules hyaline to orange or brown, walls lined by palisades of densely clustered, lageniform to cylindrical, often basally curved phialides, $6.3-13.2 \times 1.9-3.3 \mu \mathrm{m}(\overline{\mathrm{x}}=9.5, \mathrm{SD} \pm 1.5 \mu \mathrm{m} \times \overline{\mathrm{x}}=2.7, \mathrm{SD} \pm 0.3$ $\mu \mathrm{m}, \mathrm{n}=50), 1 / \mathrm{w}=(2.8-) 3.1-4.7(-6.5)$, with broad collarette, originating from large brown incrusted cells $8-15 \mu \mathrm{m}$ diam. and hyaline cylindrical cells. Conidia $2.1-3.4 \times 1.4-2.2 \mu \mathrm{m}(\overline{\mathrm{x}}=2.7$, $\mathrm{SD} \pm 0.4 \mu \mathrm{m} \times \overline{\mathrm{x}}=1.7, \mathrm{SD} \pm 0.2 \mu \mathrm{m}, \mathrm{n}=50), 1 / \mathrm{w}(1.3-) 1.4-1.9(-2.1)$, oblong to bullet-shaped or subglobose, 1-celled, hyaline, orange-brown in mass, with rounded upper and truncate lower end.
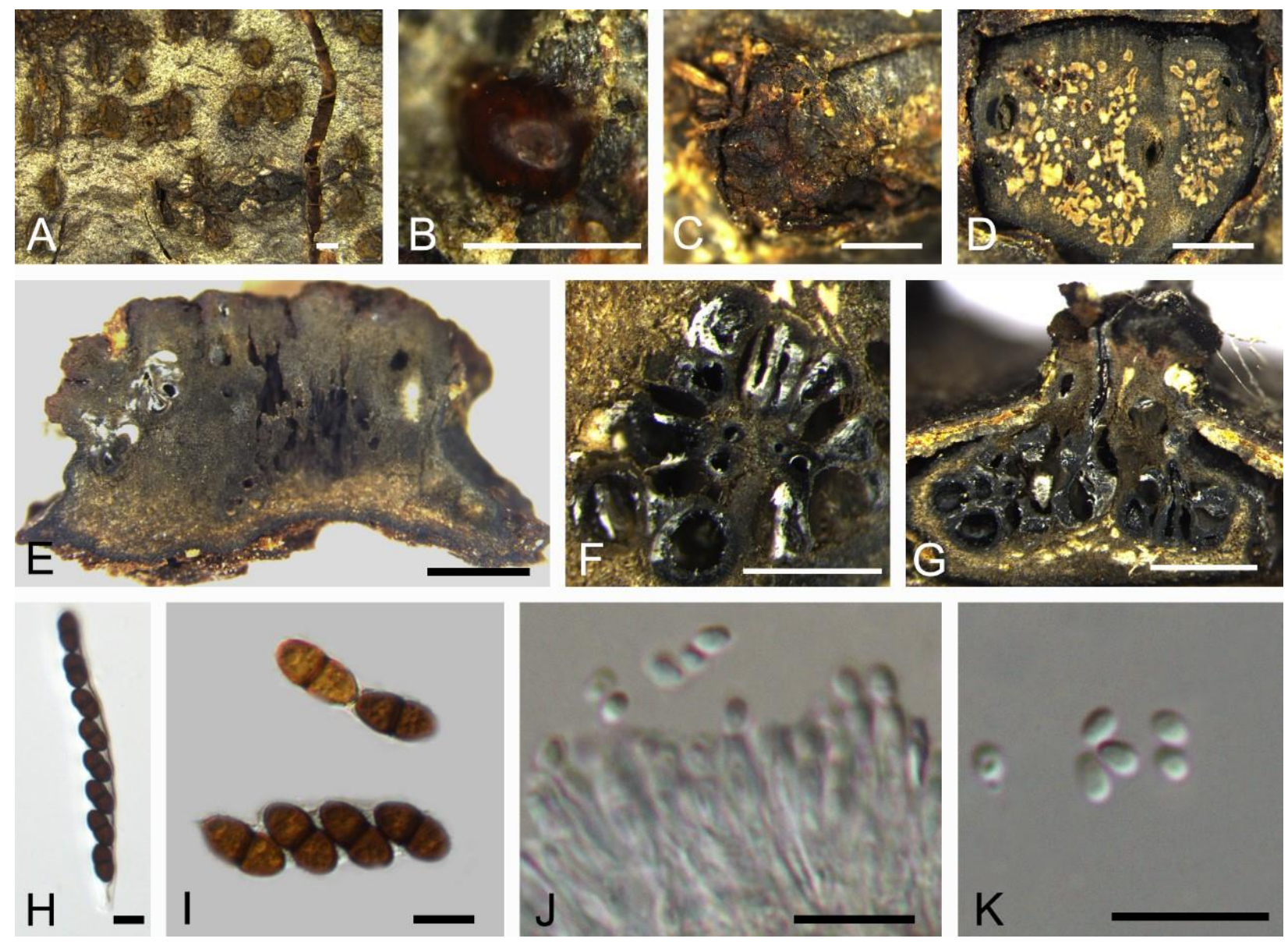

Figure 10 - Morphology of Myrmaecium fulvopruinatum from Castanea mollissima (BJFCS1450). A Habit of Stromata on the bark. B Conidiomata. C Ascomata. D Transverse sections through conidiomata. E Longitudinal sections through conidiomata. F Transverse sections through ascomata. G Longitudinal sections through ascomata. $\mathrm{H}-\mathrm{I}$ Asci and ascospores. J-K Conidiogenous cells and conidia. Scale bars: $\mathrm{A}-\mathrm{E}, \mathrm{G}=1 \mathrm{~mm}, \mathrm{~F}=0.5 \mathrm{~mm}, \mathrm{H}-\mathrm{K}=10 \mu \mathrm{m}$.

Culture characteristics - On MEA at $25^{\circ} \mathrm{C}$, colony radius $14-19 \mathrm{~mm}$ after 3 days, 26-33 mm after 5 days; centrally inoculated $90 \mathrm{~mm}$ plates entirely or nearly entirely covered by mycelium within 1 week; growth sometimes ceasing earlier. Colony zonate, surface and aerial hyphae with 
distinct macroscopically visible radial arrangement; aerial hyphae forming white radial streaks; first white, soon turning yellowish to pale orange or rosy to yellow-brown. Odour pungent, cresol-like.

Known distribution - on sun-exposed, corticated logs and branches of coniferous and broadleaf trees, worldwide, but uncommon.

Specimen examined - CHINA, Shaanxi Province, Ankang City, Xiangxidong Garden, $32^{\circ} 40^{\prime} 32.36^{\prime \prime} \mathrm{N}, 109^{\circ} 18^{\prime} 57.21 " \mathrm{E}, 1055 \mathrm{~m}$ asl, asexual morph on branches of Castanea mollissima, collected by N. Jiang, 1 July 2017 (BJFC-S1450, living culture CFCC 52769); Beijing City, Mentougou District, 39 $17^{\circ} 24.56^{\prime \prime} \mathrm{N}, 115^{\circ} 45^{\prime} 23.33^{\prime \prime E}, 452 \mathrm{~m}$ asl, sexual morph on branches of Castanea mollissima, collected by N. Jiang, 20 August 2017 (BJFC-S1451, living culture CFCC 52770); Hebei Province, Chengde City, 40²4'32.24"N, 117²8'55.24"E, $262 \mathrm{~m}$ asl, asexual morph on branches of Castanea mollissima, collected by N. Jiang, 11 October 2017 (BJFC-S1452, living culture CFCC 52771); Hebei Province, Chengde City, 40²1'44.18"N, 117²51'29.07"E, $256 \mathrm{~m}$ asl, sexual and asexual morph on branches of Castanea mollissima, collected by N. Jiang, 12 October 2017 (BJFC-S1453, living culture CFCC 52772 and CFCC 52773); Hebei Province, Chengde City, $40^{\circ} 37^{\prime} 39.14^{\prime \prime N}, 118^{\circ} 27^{\prime} 22.45^{\prime \prime} \mathrm{E}, 350 \mathrm{~m}$ asl, sexual and asexual morph on branches of Castanea mollissima, collected by N. Jiang, 14 October 2017 (BJFC-S1454, culture CFCC 52774).

Notes - Six isolates of Myrmaecium fulvopruinatum (CFCC 52769, CFCC 52770, CFCC 52771, CFCC 52772, CFCC 52773, CFCC 52774 and VFJ1) from China cluster in a wellsupported clade $(\mathrm{MP} / \mathrm{ML}=100 / 98$ ) sister to three isolates of M. fulvopruinatum (CBS 139057, CBS 139058 and CBS 139059) from Austria and America. Although strains of Myrmaecium fulvopruinatum are divided into two clades in Fig. 9, there is not obvious morphological differences among different strains in both sexual and asexual morphs, thus we identified Myrmaecium specimens from China as $M$. fulvopruinatum.

\section{Discussion}

Chinese sweet chestnut (Castanea mollissima) represents an important fruit and timber tree species, branch cankers are common fungal diseases occurring in almost all chestnut plantations in China. However, fungal species that caused canker symptoms are complex and various, for example, Cryphonectria parasitica and C. japonica share similar orange fruiting bodies on the diseased chestnut branches (Gryzenhout et al. 2009). In the present study, we carried the taxonomy work of fungi diversity on chestnut trees in China started with fungi producing melanocratic spores which are more discernible. In addition, more fungi producing hyaline spores should be solved in the future.

\section{Acknowledgements}

This study is financed by National Natural Science Foundation of China (Project No.: 31670647).

\section{References}

Alves A, Crous PW, Correia A, Phillips AJL. 2008 - Morphological and molecular data reveal cryptic speciation in Lasiodiplodia theobromae. Fungal Diversity 28, 1-13.

Carbone I, Kohn LM. 1999 - A method for designing primer sets for speciation studies in filamentous ascomycetes. Mycologia 91(3), 553-556.

Doyle JJ, Doyle JL. 1990 - Isolation of plant DNA from fresh tissue. Focus 12, 13-15.

Fan XL, Bezerra JD, Tian CM, Crous PW. 2018 - Families and genera of diaporthalean fungi associated with canker and dieback of tree hosts. Persoonia 40, 119-134.

Du Z, Fan XL, Yang Q, Hyde KD, Tian CM. 2017 - Aplosporella ginkgonis (Aplosporellaceae, Botryosphaeriales), a new species isolated from Ginkgo biloba in China. Mycosphere 8(2), $1246-1252$. 
Gryzenhout M, Wingfield BD, Wingfield MJ. 2009 - Taxonomy, phylogeny, and ecology of barkinhabiting and tree-pathogenic fungi in the Cryphonectriaceae. American Phytopathological Society Press, St Paul.

Gong S, Zhang X, Jiang S, Chen C et al. 2017 - A new species of Ophiognomonia, from northern china inhabiting the lesions of chestnut leaves infected with Diaporthe eres. Mycological Progress 16(1), 83-91.

Guindon S, Dufayard JF, Lefort V, Anisimova M et al. 2010 - New algorithms and methods to estimate maximum-likelihood phylogenies: assessing the performance of PhyML 3.0. Systematic and Biology 59(3), 307-321.

Jaklitsch WM, Fournier J, Dai DQ, Hyde KD, Voglmayr H. 2015 - Valsaria and the Valsariales. Fungal Diversity 73(1), 159-202.

Jaklitsch WM, Fournier J, Rogers JD, Voglmayr H. 2014 - Phylogenetic and taxonomic revision of Lopadostoma. Persoonia 32, 52-82.

Jami F, Slippers B, Wingfield MJ, Gryzenhout M. 2014 - Botryosphaeriaceae species overlap on four unrelated, native South African hosts. Fungal Biology 118(2), 168-179.

Jeewon R, Hyde KD. 2016 - Establishing species boundaries and new taxa among fungi: recommendations to resolve taxonomic ambiguities. Mycosphere 7(11), 1669-1677.

Jiang N, Fan XL, Yang Q, Du Z, Tian CM. 2018 - Two novel species of Cryphonectria from Quercus in China. Phytotaxa 347(3), 243-250.

Lu C, Guo SJ. 2017 - Analysis on the nutritional characters and comprehensive evaluation of 16 chestnut germplasm resources. Science and Technology of Food Industry 37, 357-376. (In Chinese)

Moncalvo JM, Wang HH, Hseu RS. 1995 - Phylogenetic relationships in Ganoderma inferred from the internal transcribed spacers and 25S ribosomal DNA sequences. Mycologia 87, 223-238.

Rigling D, Prospero S. 2018 - Cryphonectria parasitica, the causal agent of chestnut blight: invasion history, population biology and disease control. Molecular Plant Pathology 19(1), 720 .

Ronquist F, Huelsenbeck JP. 2003 - MrBayes 3: Bayesian phylogenetic inference under mixed models. Bioinformatics 19(12), 1572-1574.

Senanayake IC, Crous PW, Groenewald JZ, Maharachchikumbura SSN et al. 2017 - Families of Diaporthales based on morphological and phylogenetic evidence. Studies in Mycology 86, 217-296.

Sutton BC. 1975 - Coelomycetes. V. Coryneum. Mycological Papers 138, 1-224.

Sutton BC. 1980 - The Coelomycetes: Fungi Imperfecti with Pycnidia Acervuli and Stomata. Commonwealth Mycological Institute, Kew.

Swofford DL. 2003 - PAUP*: Phylogenetic Analysis Using Parsimony, * and Other Methods, Version 4.0b10, Sinauer Associates, Sunderland.

Tai FL. 1979 - Sylloge Fungorum Sinicorum. Science Press, Beijing.

Teng SC. 1996 - Fungi of China. Mycotaxon, Ithaca.

Tamura K, Stecher G, Peterson D, Filipski A, Kumar S. 2013 - MEGA6: molecular evolutionary genetics analysis version 6.0. Molecular Biology and Evolution 30(12), 2725-2729.

Vilgalys R, Hester M. 1990 - Rapid genetic identification and mapping of enzymatically amplified ribosomal DNA from several Cryptococcus species. Journal of Bacteriology 172(8), 42384246.

Visentin I, Gentile S, Valentino D, Gonthier P et al. 2012 - Gnomoniopsis castanea sp. nov. (Gnomoniaceae, Diaporthales) as the causal agent of nut rot in sweet chestnut. Journal of Plant Pathology 94(2), 411-419.

Voglmayr H, Akulov OY, Jaklitsch WM. 2016 - Reassessment of Allantonectria, phylogenetic position of Thyronectroidea, and Thyronectria caraganae sp. nov. Mycological Progress 15, 921.

Voglmayr H, Castlebury LA, Jaklitsch WM. 2017 - Juglanconis gen. nov. on Juglandaceae, and the new family Juglanconidaceae (Diaporthales). Persoonia 38, 136-155. 
White TJ, Bruns T, Lee S, Taylor J. 1990 - Amplification and direct sequencing of fungal ribosomal RNA genes for phylogenetics. PCR protocols: a guide to methods and applications $18,315-322$.

Wijayawardene NN, Hyde KD, Lumbsch HT, Liu JK et al. 2018 - Outline of Ascomycota: 2017. Fungal Diversity, 88(1), 167-263.

Wijayawardene NN, Hyde KD, Rajeshkumar KC, Hawksworth DL et al. 2017 - Notes for genera: Ascomycota. Fungal Diversity 86(1), 1-594.

Wijayawardene NN, Hyde KD, Wanasinghe DN, Papizadeh M et al. 2016 - Taxonomy and phylogeny of dematiaceous coelomycetes. Fungal Diversity 77(1), 1-316.

Zhang HW, Zhang GZ, Cao QC, Sun MD, Cao J. 2009 - Investigations of main kinds of pests on Chinese chestnut in Beijing. Plant Protection 35 (2), 121-124. 